\title{
Die Kartographie der Aura aus dem Geist der Wirkungsästhetik. Synästhesie und das Verhältnis von Kunst und Esoterik um 1900
}

Esoterische Bewegungen sind seit einem halben Jahrhundert in das Blickfeld der kunsthistorischen Forschung geraten. Wegweisend war der finnische Kunstwissenschaftler Sixten Ringbom (1935-1992). In seinem 1966 veröffentlichten Aufsatz Art in the ,Epoch of the Great Spiritual': Occult Elements in the Early Theory of Abstract Painting und in einer Reihe späterer Publikationen hat er versucht nachzuweisen, dass Künstler um 1910 die entscheidenden Anregungen für die „Erfindung“ Abstrakter Kunst aus der Beschäftigung mit esoterischen Schriften bezogen haben. ${ }^{1}$ Seine Untersuchungen konzentrierten sich auf Wassily Kandinsky. Andere Maler, insbesondere Franz Marc, Paul Klee und Piet Mondrian, kommen in Ringboms Schriften beiläufig vor, um die Tragweite der Argumentation besser zu verankern. Die Thesen des Kunsthistorikers aus dem südfinnischen Turku stellten die damalige Kunstgeschichte auf den Kopf und wurden anfangs nur zögerlich rezipiert, obgleich er von Ernst Gombrich, dem damaligen Direktor des Warburg Instituts, gefördert wurde. ${ }^{2}$ Seit den 1980er Jahren nimmt allerdings die Zahl von Veröffentlichungen zum Verhältnis von Esoterik und Kunst stark zu. Zeitgleich haben umfangreiche Ausstellungen diese Deutung der Abstrakten Kunst auch jenseits des Fachpublikums populär gemacht. ${ }^{3}$ Die Geburt der Abstraktion aus dem Geist

Ich danke Karl Baier, Michael Bergunder, Gerd Blum, Peter Geimer, Roland Halfen, Ulrich Kaiser und Helmut Zander für zahlreiche Anregungen, Tanja Jenni für ihre Hilfe bei den Recherchen.

1 Zusammenhänge zwischen Kandinsky und der Theosophie waren bereits zuvor in der Forschung diskutiert worden (siehe insb. Terence H. Robsjohn-Gibbings: Mona Lisa's Moustache. A Dissection of Modern Art. New York 1947). Ringbom ist aber der erste, der Kandinskys Rezeption der Theosophie eingehend untersuchte. Siehe seine Biographie und Bibliographie in: Icon to cartoon. A tribute to Sixten Ringbom. Hg. v. Marja Terttu Knapas. Helsinski 1995 (Taidehistoriallisia tutkimuksia 16), S. 9-31 u. 355-374.

2 Ebd., S. 18. Zum Verhältnis von Gombrich zu Ringbom siehe Ernst H. Gombrich: From Archaeology to Art History. Some Stages in the Rediscovery of the Romanesque. In: Icon to Cartoon (wie Anm. 1), S. 91-108.

3 Siehe insb.: Zeichen des Glaubens, Geist der Avantgarde. Religiöse Tendenzen in der Kunst des 20. Jahrhunderts. Hg. v. Wieland Schmied (Ausst.-Kat. Berlin, Schloß Charlottenburg). Stuttgart 1980; The Spiritual in Art. Abstract Painting 1890-1985. Hg. v. Maurice Tuchman u. Judi Freeman (Ausst.-Kat. Los Angeles, County Museum). Los Angeles 1986; Okkultismus und Avantgarde. Von Munch bis Mondrian 1900-1915. Hg. v. Bernhard Apke u. Ingrid Ehrhardt (Ausst.-Kat. Frankfurt a.M., Schirn Kunsthalle). Ostfildern 1995 und Das Bauhaus und die Esoterik. Johannes Itten - Wassily Kandinsky - Paul Klee. Hg. v. Christoph Wagner (Ausst.-Kat. Hamm, Gustav-Lübcke-Museum u. Würzburg, Museum im Kulturspeicher). Bielefeld, Leipzig 2005. Neben Ringboms Fokus auf die Abstraktion bilden auch die spiritistische Fotografie (Im Reich der Phantome - Fotografie des Unsichtbaren. Hg. v. Andreas Fischer. 
der Esoterik ist eine der beliebtesten Meistererzählungen zur Kunst des 20. Jahrhunderts geworden. Reinhard Zimmermann hat vor kurzem zur Frage nach den Quellen Abstrakter Kunst sogar behauptet: „Doch führt nichts an der Tatsache vorbei, dass von allen je vorgeschlagenen Bezügen und Rezeptionen diejenigen zu Theosophie und Okkultismus mit Abstand dokumentarisch am besten belegt und am deutlichsten auch in der konkreten Malweise aufgezeigt werden können." “4

Die zentralen Beweisstücke in Ringboms Argumentation sind zwei theosophische Bücher von Annie Besant und Charles Leadbeater aus den Jahren 1902 und 1905, die bemerkenswerte Farbillustrationen beinhalten (Abb. 5 u. 7-12). ${ }^{5}$ Ringbom glaubt, dass diese Bücher einen entscheidenden Einfluss auf Kandinsky ausgeübt hätten. ${ }^{6}$ Er verweist auf Kandinskys Interesse an der Theosophie und bemerkt einerseits, dass diese theosophischen Bilder ungegenständlich sind und damit die ersten abstrakten Gemälde dieses Malers vorwegnehmen, und dass sich andererseits Kandinskys Intentionen mit denen von Besant und Leadbeater insofern decken, als auch er den Anspruch erhebt, geistige Wirklichkeiten darzustellen. Darüber hinaus betont Ringbom den Stellenwert der Vibrationstheorie: Für Kandinsky und die Theosophen bestehen Farben und Töne letztlich aus gleichwertigen Vibrationen. Es gibt allerdings genauso gute Gründe, Ringboms Urteil in Frage zu stellen: Erstens sind Kandinskys Gemälde zwar wie die Illustrationen dieser theosophischen Bücher ungegenständlich, jedoch sehr verschieden von diesen eher schematischen Darstellungen. Eine direkte Anlehnung, etwa die Übernahme einzelner Formen, hat nicht stattgefunden. Zweitens ist die herausragende Bedeutung, die Ringbom Besant und Leadbeater als den Erfindern abstrakter Bilder zuweist, nicht haltbar. Zahllose ungegenständliche Bilder sind in Künstlerateliers und Kunsttraktaten des 18. und 19. Jahrhunderts unabhängig von esoterischen Zusammenhängen nachzuweisen (Abb. 1 ). ${ }^{7}$ Diese Tatsache war Ringbom nicht unbe-

Ostfildern-Ruit 1997; Le troisième oeil. La photographie et l'occulte. Hg. v. Clément Chéroux. Paris 2004) sowie der ikonographische Niederschlag der Esoterik, etwa der Theosophie auf Max Beckmann, weitere Forschungsschwerpunkte in diesem Bereich (Friedhelm Willhelm Fischer: Max Beckmann. Symbol und Weltbild. Grundriss zu einer Deutung des Gesamtwerkes. München 1972).

4 Reinhard Zimmermann: Von der Romantik zur Abstraktion? Die Esoterik und die historischen Grundlagen der abstrakten Kunst. In: Esoterik am Bauhaus. Eine Revision der Moderne? Hg. v. Christoph Wagner. Regensburg 2009, S. 55-72, hier S. 66.

5 Charles Webster Leadbeater: Man Visible and Invisible. Examples of Different Types of Men as Seen by Means of Trained Clairvoyance. London 1902; Annie Besant, Charles W. Leadbeater: Thought-Forms. London u.a. 1905, letzteres bei Ringbom irrtümlich mit Datum 1901 (Sixten Ringbom: Art in ,the Epoch of the Great Spiritual'. Occult Elements in the Early Theory of Abstract Painting. In: Journal of the Warburg and Courtauld Institutes 24 (1966), S. 386-418, hier S. 397).

6 ,[T]hese colour illustrations can be regarded as the first non-objective representations [...] they must have appeared highly suggestive to Kandinsky, whose artistic vocabulary seems to have been influenced by the occult pictorial idiom" (Ringbom: Art [wie Anm. 5], S. 404f.).

7 Für einen Überblick über die Kontexte solcher Bilder und zahlreiche Beispiele siehe Raphael Rosenberg: Turner - Hugo - Moreau. Entdeckung der Abstraktion. München 2007. 
kannt, er hat aber versucht, sie herunterzuspielen. ${ }^{8}$ Drittens spricht die Quellenlage dafür, dass Kandinsky von den Schriften Besants und Leadbeaters wenig beeindruckt war. Der Maler zitiert in seinem im Dezember 1911 veröffentlichten Buch Über das Geistige in der Kunst mehrfach Blavatsky und Steiner, nicht aber Besant und Leadbeater. In seinem Nachlass sind ausführliche Notizen zu Schriften von Steiner erhalten, Anmerkungen zu Besant oder Leadbeater konnten dagegen bis heute nicht nachgewiesen werden. ${ }^{9}$

Sixten Ringbom kommt das Verdienst zu, auf die Nähe von Esoterik und Kunst um 1900 hingewiesen zu haben. Seine Konstruktion eines Abhängigkeitsverhältnisses - der Abstrakten Kunst von der Esoterik - ist allerdings, wie es hier zu zeigen gilt, eine Fehldeutung. Die Ursache dieser Fehldeutung liegt vor allem in der Asymmetrie von Ringboms Analyse. Während er Kandinsky historisch-kritisch untersucht, fragt er nicht nach dem Kontext und nach den Grundlagen jener Theosophen, von denen er behauptet, sie wären die Quelle der Abstrakten Kunst gewesen. Diese Asymmetrie versuche ich mit dem vorliegenden Beitrag auszugleichen. Eine Untersuchung der gedruckten Schriften zeigt, dass seit dem ausgehenden 19. Jahrhundert führende Theosophen im Rahmen ihrer synkretistischen Vorgehensweise vielfach auch Anregungen aus der Kunst und Kunsttheorie geschöpft haben, vor allem aus wirkungsästhetischen Theorien, die in der Aufklärung wurzeln und um 1900 in fortschrittlichen Kunstschulen diskursbestimmend waren. Künstler wie Kandinsky fanden dadurch in der Theosophie Gedanken vor, die ihnen aus der eigenen Ausbildung vertraut waren.

8 Erst im Epilog seines Buches kommt Ringbom auf die von Otto Stelzer gesammelten Beispiele früher ungegenständlicher Bilder zu sprechen (Sixten Ringbom: The Sounding Cosmos. A Study in the Spiritualism of Kandinsky and the Genesis of Abstract Painting. Abo 1970, S. 207-210; Otto Stelzer: Die Vorgeschichte der abstrakten Kunst. Denkmodelle und VorBilder. München 1964). Er verbindet sie mit den Thesen von Klaus Lankheit und versucht, diese Werke durch die Konvergenz des romantischen und des esoterischen Spiritualismus in sein Erklärungsmodell zu integrieren (Klaus Lankheit: Die Frühromantik und die Grundlagen der ,gegenstandslosen“ Malerei. In: Neue Heidelberger Jahrbücher [1951], S. 55-90). Siehe dazu Peter Anselm Riedl: Kandinsky und die Tradition. In: Heidelberger Jahrbücher 22 (1978), S. 3-17 und Peter Anselm Riedl: Wassily Kandinsky in Selbstzeugnissen und Bilddokumenten. Reinbek bei Hamburg 1983, bes. S. 51f.

9 Ringbom hat die Notizen publiziert, die Kandinsky bei der Lektüre von Steiner machte (Sixten Ringbom: Kandinsky und das Okkulte - Die Steiner-Annotationen Kandinskys. In: Kandinsky und München. Begegnungen und Wandlungen 1896-1914. Hg. v. Armin Zweite. München 1982, S. 85-105, hier S. 87). Über die Rezeption von Besant und Leadbeater ist lediglich bekannt, dass er sie in Briefen der 1920er Jahre erwähnt (siehe Willi Grohmann: Wassily Kandinsky. Leben und Werk. Köln 1958, S. 41). 


\section{Blavatsky: Synästhesie als höhere Erkenntnis}

Helmut Zander hat gezeigt, dass esoterische Bewegungen der Zeit um 1900 auf Diskursstränge zurückgehen, die die Etablierung der modernen Naturwissenschaft begleiten. Die Erfindung des Fernrohrs im frühen 17. Jahrhundert ermöglichte eine genauere Beobachtung von Planeten und Sternen. Die Erkenntnis, dass der Mond von Kratern übersät sei, aber keinerlei Spuren von Seelen und Göttern zeigt, führte zu einer tiefgreifenden Desillusionierung. Der metaphysische Himmel wurde zum physischen Weltall degradiert. Emanuel Swedenborg (1688-1772), Friedrich Christoph Oetinger (1702-1782), Gotthold Ephraim Lessing (1729-1781) und viele andere haben als Reaktion auf diese durch Sehhilfen forcierte Entweihung des Kosmos Theorien einer , höheren Erkenntnis' entwickelt. Grundidee dieser Theorien ist, dass es dem Menschen unter bestimmten Bedingungen möglich sei, das metaphysische Jenseits mit höheren, d.h. über-sinnlichen Organen wahrzunehmen. Der ,Seher', der mit solchen Organen ausgestattet ist, ,sieht ' das Jenseits genauso wie der Astronom mit seinem Fernrohr die Krater des Mondes beobachtet. Diese Ansätze sollten das Objekt der (alten) Theologie mit dem empirischen Anspruch der (neuen) Naturwissenschaften in Einklang bringen. Phänomene, die man beobachten, aber naturwissenschaftlich nicht erklären konnte, rückten damit in den Mittelpunkt esoterischer Bewegungen; ${ }^{10}$ dazu zählen der Magnetismus, die Hypnose, aber auch, was bislang übersehen wurde, die Synästhesie.

Synästhesie bezeichnet die Verknüpfung der Sinne. Das Wort wurde 1864 geprägt und hat sich im Verlauf des 20. Jahrhunderts eingebürgert. ${ }^{11}$ Es handelt sich um eine nicht allzu seltene Anomalie der Sinneswahrnehmung, ${ }^{12}$ bei der es anlässlich der „Stimulation einer Sinnesqualität - beispielsweise des Hörens oder des Riechens - zusätzlich in einer anderen Sinnesqualität, wie dem Sehen von Farben oder von geometrischen Figuren, zu einer Sinneswahrnehmung kommt“. So die Definition des Psychiaters und Neurologen Hinderk Emrich, der ferner schreibt:

Am häufigsten ist dabei das so genannte ,farbige Hören ' - auch als Farbenhören, ,Audition colorée“ und ,coloured hearing ' bezeichnet. Dabei führen Geräusche, Musik, Stimmen, ausgesprochene Buchstaben und Zahlen typischerweise zur Wahrnehmung bewegter Farben und

10 Helmut Zander: Höhere Erkenntnis. Die Erfindung des Fernrohrs und die Konstruktion erweiterter Wahrnehmungsfähigkeiten zwischen dem 17. und dem 20. Jahrhundert. In: Trancemedien und Neue Medien um 1900. Hg. v. Markus Hahn u. Erhard Schüttelpelz. Bielefeld 2009, S. 17-55.

11 Ludwig Schrader: Sinne und Sinnesverknüpfungen. Studien und Materialien zur Vorgeschichte der Synästhesie und zur Bewertung der Sinne in der italienischen, spanischen und französischen Literatur. Heidelberg 1969, S. 46-55.

12 Die Häufigkeit von Synästhesie ist umstritten. Neueste Studien schätzen, dass zwei bis vier Prozent der Bevölkerung davon betroffen sind. In einer Studie mit Zürcher Kunststudenten waren es sieben Prozent (Nicolas Rothen u. Beat Meier: Higher Prevalence of Synaesthesia in Art Students. In: Perception 39/5 [2010], S. 718-720). Die Anlage scheint in hohem Maße vererbbar zu sein (Kylie J. Barnett u.a.: Familial Patterns and the Origins of Individual Differences in Synaesthesia. In: Cognition 106/2 [2008], S. 871-893). 
Formen. Sie werden von den Betroffenen in die Außenwelt oder auch ins Kopfinnere projiziert. Auf einem ,inneren Monitor', der allerdings keine räumliche Begrenzung aufweist, erscheinen dann meist vorbeilaufende farbige Strukturen, Kugeln oder langgestreckte vorüberziehende dreidimensionale Gebilde mit charakteristischen Oberflächen, beispielsweise samtigen, glitzernden oder auch gläsernen oder metallischen Flächen. ${ }^{13}$

Mir sind keine interkulturellen empirischen Studien über Synästhesie bekannt. Nach dem derzeitigen Stand der Forschung darf aber angenommen werden, dass das Phänomen unabhängig von Erziehung und Kultur entsteht, wahrscheinlich in Verbindung mit spezifischen Schaltungen zwischen bestimmten Gehirnarealen. ${ }^{14}$ Synästhesie dürfte also ein von soziohistorischen Rahmenbedingungen relativ unabhängiges Phänomen sein, auch wenn es erst spät beschrieben wurde. Die ersten Erwähnungen datieren aus der Zeit um 1700, ${ }^{15}$ eine vertiefte Auseinandersetzung beginnt in den 1870er Jahren, die ältesten systematischen Abhandlungen entstehen in den 1880er und 1890er Jahren und stammen vielfach aus der Feder führender Psychologen bzw. Psychiater wie Francis Galton (1822-1911), Théodore Flournoy (1854-1920) und Eugen Bleuler (1857-1937). ${ }^{16}$ Zwischen den 1880er und 1930er Jahren erlebt die Synästhesieforschung einen Boom (Tab. 1). Allein im Jahr 1893 erscheinen 24 Titel, genauso viele wie zwischen 1786 und 1863 zusammengenommen. ${ }^{17}$ Die sich damals etablierende experimentelle Psychologie versuchte, Wahrnehmung und Denken wissenschaftlich zu erfassen und die Bandbreite menschlicher Fähigkeiten und Reaktionen zu quantifizieren. In diesem Kon-

13 Hinderk M. Emrich, Udo Schneider u. Markus Zedler: Welche Farbe hat der Montag? Synästhesie. Das Leben mit verknüpften Sinnen. Stuttgart u.a. 2002, S. 11f. Zur Synästhesie um 1900 vgl. auch den Beitrag von Jürgen Stolzenberg im vorliegenden Band.

14 Edward M. Hubbard u. Vilayanur S. Ramachandran: Neurocognitive Mechanisms of Synesthesia. In: Neuron 48/3 (2005), S. 509-520 und das Sonderheft: Synaesthesia, Journal of Neuropsychology 5/2 (2011), S. 145-371.

15 Albert Wellek: Farbenharmonie und Farbenklavier. Ihre Entstehungsgeschichte im 18. Jahrhundert. In: Archiv für die gesamte Psychologie 94 (1935), S. 347-375, hier bes. S. 349f.

16 Eugen Bleuler u. Karl Lehmann: Zwangsmässige Lichtempfindungen durch Schall und verwandte Erscheinungen auf dem Gebiete der anderen Sinnesempfindungen. Leipzig 1881; Francis Galton: Inquiries into Human Faculty and its Development. London 1883; Théodore Flournoy: Des phénomènes de synopsie (audition colorée): photismes, schèmes visuels, personnifications. Paris, Genève 1893.

17 Friedrich Mahling hat die bislang umfangreichste Geschichte der Synästhesieforschung verfasst (Friedrich Mahling: Das Problem der, Audition colorée'. Eine historisch-kritische Untersuchung. In: Archiv für die gesamte Psychologie 57 [1926], S. 165-301). Meine Tabelle ergänzt seine bis 1926 sehr vollständige Bibliographie mit Daten aus PsycInfo (abgerufen am 02.05.2011). Eine vergleichbare Aufstellung von John Harrison ist sehr unvollständig (John Harrison: Wenn Töne Farben haben. Synästhesie in Wissenschaft und Kunst. Heidelberg 2007, S. 27). Beide zeigen jedoch, dass seit den 1930er Jahren das Interesse an der Synästhesie abrupt nachgelassen hat, da diese im Kontext des neuen behavioristischen Paradigmas uninteressant wurde. Seit den 1990er Jahren gibt es ein erneutes experimentelles Interesse an Synästhesie, diesmal unter dem Vorzeichen der Neurologie: Man versucht anhand bildgebender Messungen von Gehirnaktivität die Verknüpfung der Sinnesbereiche zu erklären und hofft, dass über die Synästhesie hinaus Erkenntnisse über die Funktion des Gehirns gewonnen werden können. 
text war das Phänomen der Synästhesie interessant, weil es am Rand der Normalität angesiedelt ist und weil es Rätsel aufwirft, die als Herausforderung für diese junge Wissenschaft angesehen wurden. Ähnliches gilt für Erscheinungen, die später unter dem Begriff der ,Parapsychologie" zusammengefasst wurden. ${ }^{18}$ Die Biographie des Schweizers Théodore Flournoy, der 1893 ein erstes umfangreiches Handbuch über Synästhesie verfasst hat, ist charakteristisch für diese Ausrichtung der neuen Disziplin. 1878/79 studiert Flournoy bei Wilhelm Wundt in Leipzig, während dieser das erste Labor für experimentelle Psychologie aufbaut. 1891 begründet er den Lehrstuhl für Psychologie an der Universität Genf, den weltweit ersten, der an einer naturwissenschaftlichen Fakultät angesiedelt ist. Damit einhergehend richtet Flournoy 1892 das erste Labor für experimentelle Psychologie in der Schweiz ein. ${ }^{19}$ Sein grundlegendes Buch über Synopsien (1893) zeigt, wie sehr sich die Einstellung zur Synästhesie in dieser Zeit verändert. Während man bis in die 1860er Jahre hinein die ,audition colorée‘ als Pathologie wahrgenommen hatte, etwa als ein zu viel an Farben polar zur Farbblindheit, ${ }^{20}$ versteht Flournoy diese als eine ,Gabe' und bedauert, sie nicht selbst zu besitzen. ${ }^{21}$ In den Jahren nach den Studien über Synästhesie widmet sich Flournoy mit einem unverändert hohen wissenschaftlichen Anspruch spiritistischen Phänomenen. ${ }^{22}$

Théodore Flournoy ist nicht der einzige Naturwissenschaftler, der sich um 1900 für ,esoterische' Phänomene interessiert. Seine Biographie zeigt, dass die Grenze zwischen Esoterik und Wissenschaft im ausgehenden 19. Jahrhundert durchlässiger war als heute. Die Akteure lassen sich zwar deutlich unterscheiden, sowohl auf Grund ihres methodischen Vorgehens - Experiment und Quellenkritik auf der einen, Hellsehen und unkontrollierte Ereignisse wie spiritistische Sitzungen auf der anderen Seite - wie auch durch ihre institutionelle Einbindung: öffentliche Universitäten versus private Gesellschaften. Beide Seiten interessieren sich aber für dieselben Phänomene und konkurrieren um deren Deutungshoheit. Dabei ist es auffällig, dass in dieser Zeit auf der Seite der Esoterik der Ruf nach einem wissenschaftlichen Vorgehen steigt und es seitens der Naturwissenschaften keine Scheu gibt, esoterische Praktiken anzuwenden. So hat beispielsweise Jean-Martin Charcot

18 Auf die Verwandtschaft von Synästhesie und Vision hat bereits Karl Clausberg hingewiesen und in diesem Zusammenhang - ausgehend von Walter Benjamin - auch die Aura, etwa die Migräne-, Haschisch- und Kunst-Aura, kulturgeschichtlich diskutiert. Explizit lässt er dabei allerdings die ,theosophisch-spiritistischen Modeströmungen“ aus (Karl Clausberg: Aura als Ausdruck - Synästhesien der Beseelung. In: Ausdruck, Ausstrahlung, Aura. Synästhesien der Beseelung im Medienzeitalter. Hg. v. Karl Clausberg. Bad Honnef 2007, S. 41-86, hier S. 71).

19 Serge Nicolas, Agnes Charvillat: Théodore Flournoy (1854-1920) and Experimental Psychology. Historical Note. In: The American Journal of Psychology 111/2 (1998), S. 279_ 294, hier S. 280-283.

20 So Charles Aug. Édouard Cornaz: Des abnormités congéniales des yeux et de leurs annexes. Lausanne 1848, S. 150, zit. nach Mahling: Audition colorée (wie Anm. 17), S. 190, der die Veränderung der Einstellungen detailliert nachzeichnet.

21 Flournoy: Synopsie (wie Anm. 16), S. 2.

22 Nicolas, Charvillat: Flournoy (wie Anm. 19), S. 288-290. 
(1825-1893), Chef-Arzt der Pariser Salpêtrière und weltweit erster Inhaber eines Lehrstuhls für Neurologie, die Hypnose nicht nur als Objekt wissenschaftlicher Studien betrachtet, sondern auch als Mittel der Therapie eingesetzt, und man vergisst gerne, dass Hypnose an deutschen Universitäten bis in die 1950er Jahre von Psychiatern gelehrt und praktiziert wurde (etwa zur Behandlung von Enuresis). ${ }^{23}$

Die 1875 in New York gegründete Theosophische Gesellschaft ist um die Jahrhundertwende die international erfolgreichste esoterische Bewegung. Leitfigur und Mitbegründerin dieser Gesellschaft war Helena Petrovna Blavatsky (1831-1891), die Theosophie als eine den Naturwissenschaften überlegene, okkulte Wissenschaft definiert: ${ }^{24}$ „The true occultist is acquainted with no single problem that esoteric science is unable to solve." Blavatsky legt dar, dass die ,akademische“, ,westliche" 25 Wissenschaft nur die physische Realität berücksichtige, während der Theosoph auch das einbeziehe, was dem bloßen Auge unzugänglich sei, das Psychische und das Geistige. In einem Aufsatz mit dem programmatischen Titel Occult or Exact Science? gibt Blavatsky 1886 verschiedene Beispiele von Phänomenen, die sich dem Zugriff der Naturwissenschaften entziehen, während sie von der Theosophie erklärt werden können. Aufschlussreich ist, dass sie dabei zuerst und am ausführlichsten die Synästhesie diskutiert. ${ }^{26}$ Sie berichtet von einem Mädchen, das Klänge mit Farben assoziierte und beim Klavierspiel der Mutter beglückende Regenbogen sah. Die besorgten Eltern ließen einen Arzt kommen, der dem Mädchen mehr physische Bewegung verschrieb. Blavatsky behauptet aber, dass das Mädchen keineswegs krank, sondern ,sensitiv“ sei: Es besitze die Gabe übersinnlicher Wahrnehmung. ${ }^{27}$

Die Instrumentalisierung der Synästhesie durch die Theosophie war in zweierlei Hinsicht vielversprechend. Einerseits diente Synästhesie als Exempel, um die Überlegenheit der Theosophie gegenüber der akademischen Wissenschaft zu beweisen. Blavatsky tritt mit dem Anspruch auf, ein Phänomen zu erklären, dessen Ursachen der universitären Wissenschaft verborgen bleiben. Auf der anderen Seite lieferte diese Erklärung den für die Theosophie entscheidenden Beweis dafür, dass es jenseits des Sichtbaren eine Wirklichkeit gibt, die von manchen Menschen, also etwa von denen, die eine theosophische Schulung durchlaufen haben, wahrgenommen werden kann. Im Rahmen dieses Aufsatzes geht Blavatsky allerdings nicht ins Detail. Der Leser erfährt nichts darüber, was der Synästhetiker sieht,

23 Steffen Böhm u.a. zeigen am Beispiel der Entstehung der Psychoanalyse, wie es nur sehr allmählich zu einer schärferen Grenzziehung kam (Steffen Böhm u.a.: Verdrängte Ursprünge. In: Jahrbuch für Universitätsgeschichte 12 [2009], S. 13-39).

24 Helena Petrovna Blavatsky: Occult or Exact Science? In: The Theosophist 7/79 (1886), S. 422-431 u. 481-494, zit. nach ders.: Collected Writings. Bd. 7. 3. Aufl. Madras u.a. 1987 [zuerst 1959], S. 55-90, hier S. 55.

25 Blavatsky verwendet die Bezeichnungen „Exact Science“ und „Western Science“ als Synonyme (ebd., S. 78).

26 Ebd., S. 56-68.

27 Ebd., S. 57 u. 64. 
welche höheren Schichten des Daseins er wahrnimmt. Offensichtlich hat sie sich zu diesem Zeitpunkt noch kaum Gedanken darüber gemacht. Das liegt aber auch daran, dass die Begründerin der Theosophischen Gesellschaft zwischen öffentlich verfügbarem, ,exoterischem" und geheimem, ,esoterischem' Wissen trennt. Was ,Sensitive" durch ihre höheren Wahrnehmungsorgane sehen, beschreibt sie in Texten, die an die Esoteric Section of the Theosophical Society adressiert sind, einen 1888 gegründeten, exklusiven inneren Schülerkreis. ${ }^{28}$ Dort heißt es, dass Synästhetiker die übersinnliche Aura, die eiförmig jeden Menschen umgibt, wahrnehmen. Demnach verraten die Farben und Formen dieser Aura dem Seher, was dem gewöhnlichen Auge verborgen bleibt - seinen Charakter, seine geistige Entwicklung, seine Gefühle und Gedanken:

Not only Adepts and advanced Chelas, but also the lower order of psychics, such as clairvoyants and psychometrists, can perceive a psychic Aura of various colors around every individual, corresponding to the temperament of the person within it. In other words the mysterious records within the Auric Egg are not the heirloom of trained Adepts alone, but sometimes also of natural psychics. Every human passion, every thought and quality, is indicated in the Aura by corresponding colors and shades of color, and certain of these are sensed and felt rather than perceived. The best of such psychics, as shown by Galton, can also perceive colors produced by the vibrations of musical instruments, every note suggesting a different color. ${ }^{29}$

Die Aura ist keine theosophische Erfindung. Die Vorstellung, dass eine mehr oder weniger unsichtbare Hülle den Menschen umgibt, reicht bis in die Antike zurück. Ältere Vorstellungen, etwa im Christentum ${ }^{30}$ und im Buddhismus, ${ }^{31}$ gehen von einer Lichtsphäre als Auszeichnung besonderer Menschen aus - dem Nimbus der Heiligen oder die Mandorla Christi. Die demokratische Vorstellung, wonach alle Menschen eine Aura besitzen, erscheint erst später. ${ }^{32}$ Eine systematische Ge-

28 Zur Geschichte der Esoteric Section siehe Henk J. Spierenburg: The Inner Group Teachings of H. P. Blavatsky to her Personal Pupils, 1890-91. San Diego 1996, S. vii-xxviii.

29 Helena Petrovna Blavatsky: Collected Writings. 15 Bde. Wheaton, Ill. u.a. 1972-2002, hier Bd. 12. 1987, S. 621. Es handelt sich um die dritte Instruction für die esoterische Schule, ein Text, der 1889-90 entstand. Siehe auch ebd., S. 565.

30 Arnold Angenendt: Heilige und Reliquien. Die Geschichte ihres Kultes vom frühen Christentum bis zur Gegenwart. München 1997, S. 115-119.

31 Deborah Klimburg-Salter u. Maurizio Taddei: The Usnisa and the Brahmarandhra: An Aspect of Light Sybolism in Gandharan Buddha Images. In: Aksayanivi. Hg. v. Gouriswar Bhattacharya. New Delhi 1991, S. 73-93.

32 Karl Baier hat mich darauf aufmerksam gemacht, dass diese Vorstellung in mesmeristischen Kreisen verbreitet ist. So schreibt Jung-Stilling 1808: „Im natürlichen Zustand ist die Menschenseele unsichtbar; die magnetisch Schlafenden aber sehen sie wie einen himmelblauen Lichtschimmer, der den ganzen Körper auf eine gewisse Weise umgiebt, so daß also jeder Mensch einen seelischen Dunstkreis um sich her hat [...]“ (Johann Heinrich Jung-Stilling: Theorie der Geister-Kunde in einer natur-, vernunft- und bibelmäsigen Beantwortung der Frage: Was von Ahnungen, Gesichten und Geistererscheinungen geglaubt und nicht geglaubt werden müße. Nürnberg 1808, S. 62). Carl Kluge berichtet von Somnambulen, die das elektrische Fluidum von Magnetiseuren in verschiedenen Formen und Dynamiken, aber auch mit teils wechselnden Farben wahrnehmen, abhängig vom Material, durch das es fließt (Carl Alexander Ferdinand Kluge: Versuch einer Darstellung des Magnetismus als Heilmittel. Berlin 
schichte der Vorstellungen von Aura steht noch aus, Blavatsky dürfte darin eine wichtige Rolle spielen. Ihre Verknüpfung von Aura und Synästhesie ist, soweit ich sehe, gänzlich originell, und sie hatte tiefgreifende Folgen - weniger für das Verständnis der Synästhesie als für dasjenige der Aura. Durch die Angleichung mit den Erfahrungen der Synästhetiker öffnete Blavatsky den Weg, die Aura mit einer differenzierten Vielfalt charakteristischer Farben und Formen zu beschreiben.

\section{Leadbeater und Besant: Die Kartographie der Aura aus dem Geist der Kunsttheorie}

Blavatsky behauptet also, dass der Synästhetiker und der mit übersinnlichen Organen ausgestattete Eingeweihte Auren sehen, dass sie auf Grund ihrer Farbnuancen Emotionen und Gedanken wahrnehmen können. Was aber ,sehen' sie dabei genau? Was bedeuten die einzelnen Farben? Die 1891 verstorbene Gründerin der Theosophie hat nur vereinzelte und äußerst summarische Aussagen darüber getroffen. ${ }^{33}$ Nach ihrem Tod ist das Thema von ihren Adepten intensiv diskutiert worden. Führende Figuren der Theosophischen Gesellschaft haben wiederholt Publikationen beigesteuert. Wichtig für die Ausbildung einer anschaulichen Vorstellung von Auren waren insbesondere Alfred Percy Sinnett (1840-1921), der zeitweilige Vizepräsident der Theosophischen Gesellschaft Adyar, Annie Besant (1847-1933), die nach Auseinandersetzungen und Spaltungen die Leitfigur dieser internationalen Bewegung wurde, und Charles Leadbeater (1847-1934). Leadbeater zählte zwar nicht zum engeren Schülerkreis von Blavatsky, der Inner Group der Esoteric Section, ${ }^{34}$ und nahm auch später keines der höchsten Ämter in der Gesellschaft ein. Er sprach aber offensiver als andere Theosophen über eigene übersinnliche Erfahrungen und war mehrere Jahrzehnte lang aufs Engste mit Besant verbunden. Man gewinnt den Eindruck, dass er sie mit esoterischem Wissen versorgte und sich damit unentbehrlich gemacht hat. ${ }^{35}$

Sinnett hat 1893 einen Vortrag mit dem Titel The Human Aura veröffentlicht. Darin versucht er nach eigenen Angaben anhand der Aussagen verschiedener Hellseher („clairvoyants“) die spärlichen Hinweise von Blavatsky zu erweitern. Erst-

1815, S. 118-122). Blavatskys älteste Äußerungen zur Aura (ab 1880) zeigen, dass sie von einer magnetischen Aura im Sinne Mesmers ausgeht (siehe Lemma „Aura[s]“ versus das erst in späteren Schriften verwendete „Auric Egg [or Envelope]“ im Index von Blavatsky: Collected Writings [wie Anm. 29]. Bd. 15. 1991, S. 45f.).

33 Blavatsky: Collected Writings (wie Anm. 29). Bd. 12, S. 565-567. Siehe auch Spierenburg: Inner Group (wie Anm. 28), passim und William Quan Judge: The Culture of Concentration. In: The Path. A Magazine Devoted to the Brotherhood of Humanity 3 (1888), zit. nach der Onlinepublikation (URL: http://hpb.narod.ru/CultureConcentration.htm [27.01.2010]).

34 Sein Name taucht in den von Spierenburg publizierten Listen nicht auf (Spierenburg: Inner Group [wie Anm. 28], S. xiii-xxxiii).

35 Vgl. Anne Taylor: Annie Besant. A Biography. Oxford u.a. 1992, S. 282, 290 u. 294. 
mals beschreibt er die Bedeutung einzelner Farben, etwa die Auswirkung eines Zornesausbruchs: ,an outburst of anger will charge the whole aura with deep red flashes on a dark ground, while sudden terror will in a moment change everything to a mass of ghastly livid grey." Derart konkrete Angaben kommen in dem zwanzig Seiten langen Text allerdings nur vereinzelt vor, am ehesten über die Farbe Rot: ,[R]ed of one kind may indicate anger of a brutal and selfish type, red of another tint may represent what would more correctly be called, noble indignation", while other more delicate tints of red, again, are directly associated with the emotion of love, even in its loftier and purer aspect." Sinnett schreibt ferner: „The purpose of this publication scarcely require me to attempt anything like a complete catalogue of the various colours that may be discerned in auras, with the special significance attached to each." 36

Es dauerte nur zwei Jahre, bis Leadbeater einen solchen Katalog der Farben und ihrer Bedeutung in einem Aufsatz mit dem schlichten Titel The Aura publizierte. ${ }^{37}$ Der Text ist in der Zeitschrift The Theosophist wie auch als Sonderdruck mit einem Vorwort von Henry Steel Olcott, dem damaligen Präsidenten der Theosophischen Gesellschaft, erschienen. ${ }^{38}$ Bei dem selbstständigen Druck wird der Artikel mit einem erklärenden Untertitel versehen: An Enquiry Into the Nature and Functions of the Luminous Mist Seen About Human and Other Bodies. Diese Erläuterung zeigt, dass sich der Begriff Aura selbst in theosophischen Kreisen noch nicht eingebürgert hatte. Mit dem Begriff Enquiry macht Leadbeater deutlich, dass er, ganz im Sinne Blavatskys, Theosophie als eine empirische Wissenschaft, als Erforschung des Übersinnlichen versteht. Entsprechend gibt er vor, Ergebnisse von Untersuchungen darzustellen, die über längere Zeit von unabhängigen Betrachtern gemacht wurden: „It is the result of a series of investigations pursued for some years by several independent trained observers. “" ${ }^{39}$ Leadbeater erläutert, dass Auren nicht nur an Menschen beobachtet werden, sondern auch bei Tieren, Bäumen und Gesteinen. ${ }^{40}$ Er geht davon aus, dass die Aura in sieben aufsteigende Ebenen geteilt ist, von denen bis zu fünf sichtbar sind, ${ }^{41}$ wobei er sich auf den seelischen Zustand (dritte Ebene) und die Persönlichkeit (vierte Ebene) konzentriert. Obgleich Leadbeater Sinnetts Text nicht erwähnt, geht seine Abhandlung teils wörtlich auf diesen zurück. So schreibt er beispielsweise: „Deep red flashes on a black ground show anger." ${ }^{\circ 42}$

36 Alfred Percy Sinnett: The Human Aura. In: Transactions of the London Lodge of the Theosophical Society 18 (1893), S. 3-23, hier S. 16, 19 u. 21.

37 Charles W. Leadbeater: The Aura. In: The Theosophist 17 (1895), S. 134-141.

38 Charles W. Leadbeater: The Aura. An Enquiry into the Nature and Functions of the Luminous Mist seen about Human and other Bodies. Madras 1895. 1897 kam es zu einer zweiten Auflage.

39 Leadbeater: The Aura (wie Anm. 37), S. $134 \mathrm{f}$.

40 Ebd., S. 135.

41 Ebd., S. 136.

42 Ebd., S. 139. 
Im September 1896 veröffentlicht Annie Besant in dem von ihr herausgegebenen Lucifer. A Theosophical Magazine einen Aufsatz unter dem Titel Thought Forms. Sinnett und Leadbeater werden nicht erwähnt, obgleich sie die Grundlage des Textes bilden. Besant definiert Gedankenformen (thought-forms) als den Teil der Aura, in dem Seele und Geist sichtbar werden. ${ }^{43}$ Dies deckt sich mit der dritten und vierten Ebene von Leadbeater, dessen Farbpalette sie nahezu wörtlich übernimmt. Während der Text wenig innovativ ist, geht sie in einem entscheidenden Punkt über ihre Vorgänger hinaus: Sie illustriert ihren Bericht anhand von zwölf Zeichnungen, die auf vier Farbtafeln gedruckt sind (Abb. 2) ${ }^{44}$ Auch Besant agiert mit dem Gestus der experimentellen Wissenschaftlerin; die Entstehung der Bilder erläutert sie folgendermaßen:

The pictures of thought-forms herewith presented were obtained as follows: two clairvoyant Theosophists observed the forms caused by definite thoughts thrown out by one of them, and also watched the forms projected by other persons under the influence of various emotions. They described these as fully and accurately as they could to an artist who sat with them, and he made sketches and mixed colours, till some approximation to the objects was made.

Warnend vermerkt sie dabei, es handle sich um verallgemeinernde Vereinfachungen:

It may be well to remark that, in this last respect, our illustrations are a little misleading, for the thought-forms of which the air is full are far more composite than those selected as examples. These drawings represent simple thoughts and passions, of characteristic types, whereas most of those seen by the clairvoyant are exceedingly mixed. ${ }^{45}$

Annie Besant leitet mit diesem Aufsatz eine Wende der Aura-Vorstellungen ein. Wie Wilhelm Conrad Röntgen nur wenige Monate früher, im Dezember 1895, die unsichtbaren ,X-Strahlen“ durch fotografische Aufnahmen sichtbar und damit glaubwürdig gemacht hatte, verleiht sie der unsichtbaren Aura durch farbige Bilder Anschaulichkeit und damit eine höhere Überzeugungskraft. ${ }^{46}$ Die Verwendung von Illustrationen verstärkt einen Wechsel der Referenzen, der bereits in Leadbeaters Aufsatz begonnen hatte: Die Aura wird als Bild mit Begriffen der Kunst und

43 „Mental and desire bodies“ (Annie Besant: Thought-Forms. In: Lucifer. A Theosophical Magazine 19 [April 1896], S. 65-75, hier S. 68).

44 Ebd.; dieser Aufsatz, der sowohl den Titel wie auch die gesamte inhaltliche Ausrichtung des gleichnamigen Buches (Besant, Leadbeater: Thought-Forms [wie Anm. 5]) vorwegnimmt, ist in der einschlägigen Sekundärliteratur übersehen worden, so auch in Rosenberg: Abstraktion (wie Anm. 7), S. 305-307, was dazu führte, dass ich Besants Beitrag gegenüber jenem von Leadbeater unterschätzt hatte. Aufschlussreich ist, dass sich Besant auf Baraduc beruft, der bereits zuvor versucht hatte, Gedanken fotografisch, d.h. bildlich, festzuhalten (Besant: Thought-Forms [wie Anm. 43], S. 66f.).

45 Ebd., S. 67 u. 70.

46 Vgl. Helmut Zander: Rudolf Steiner. Die Biografie. München, Zürich 2011, S. 174. Röntgenbilder wurden bereits wenige Monate nach ihrer Erfindung allerorts hergestellt und reproduziert. Siehe Fotografie und das Unsichtbare 1840-1900. Hg. v. Corey Keller u.a. Wien 2009, S. 34f. u. Taf. 115-140. 
Kunsttheorie diskutiert, während okkulte bzw. theologische Kategorien zweitrangig werden. Obgleich Besant vorgibt, übersinnliche Tatsachen zu beschreiben, lehnt sich der Text formal und inhaltlich an die Analyse der Bestandteile von Bildern und deren Wirkung an. Einerseits setzt sie zentrale und etablierte Begriffe der Kunstliteratur ein. Analog zum Bild des Künstlers spricht sie von der „Herstellung“ (production) von Gedankenformen und beschreibt deren Eigenschaften, als seien es Zeichnungen eines Künstlers mit den Kategorien Farbe, Form und Umriss: „Three general principles underlie the production of all thought-forms : 1. Quality of thought determines colour. 2. Nature of thought determines form. 3. Definiteness of thought determines clearness of outline. " 47 Auf der anderen Seite sind auch die Inhalte ihrer Darstellung vielfach der Kunst und Kunsttheorie entnommen. Um bei dem bereits gegebenen Beispiel zu bleiben, sei die Stelle über die Aura eines zornigen Menschen zitiert: „Anger gives rise to red, of all shades from lurid brickred to brilliant scarlet; brutal anger (Fig. 4 [hier in Abb. 2]) will show as flashes of lurid dull red from dark-brown clouds". ${ }^{48}$ Diese Zeilen sind nahezu wörtlich aus den Aufsätzen von Sinnett und Leadbeater übernommen. Neu ist aber das Bild, in dem Zorn als rote Zickzack-Linie erscheint, die einer Wolke entspringt. Auf den ersten Blick erscheint diese Bilderfindung originell. Dass diese Darstellung des Zornes verständlich, ja geradezu einleuchtend ist, beruht allerdings auf der Verwendung von mindestens drei verschiedenen Elementen künstlerischer Diskurse. Erstens in Hinblick auf die Farbe: Rot wird in den wirkungsästhetischen Farbenlehren nicht nur als die kräftigste und aktivste Farbe, sondern häufig auch als Ausdruck innerer Aufwallungen beschrieben. So hält Giovanni Paolo Lomazzo in seinem Trattato dell'Arte de la pittura (1584) fest, dass „Rot Rache konnotiert“. ${ }^{49}$ Es ist das umfangreichste Malerei-Traktat des 16. Jahrhunderts und zugleich das erste, in dem die Wirkung der einzelnen Farben ausführlich diskutiert wird. James Usher behauptet in dieser Tradition zwei Jahrhunderte später: „Colours, like notes of music, affect the passions; red incites anger" ${ }^{60}{ }^{50}$ Und so notiert Goethe in seiner Farbenlehre von 1810:

Das angenehme heitre Gefühl, das uns das Rotgelbe noch gewährt, steigert sich bis zum unerträglich Gewaltsamen im hohen Gelbroten [...]. Die aktive Seite ist hier in ihrer höchsten Energie, und es ist kein Wunder, dass energische, gesunde, rohe Menschen sich besonders an dieser Farbe erfreuen. ${ }^{51}$

47 Besant: Thought-Forms (wie Anm. 43), S. 70f.

48 Ebd., S. 71.

49 „Il rosso che denota vendetta“ (Giovanni Paolo Lomazzo: Trattato dell'arte de la Pittura. [...] Diviso in sette libri. Ne' quali si contiene tutta la Theorica \& la prattica d'essa pittura. Milano 1584, S. 205).

50 James Usher: Clio. Or, a Discourse on Taste. Adressed to a Young Lady. The second Edition, with large Additions. Dublin 1770, S. 175.

51 Johann Wolfgang Goethe: Zur Farbenlehre. Tübingen 1810, S. 774f. 
Zweitens in Hinblick auf die Linie: Seit der Mitte des 18. Jahrhunderts entwickelt sich eine ästhetische Beschreibung der verschiedenen Linienarten. In diesem Kontext schreibt beispielsweise 1755 Moses Mendelssohn: „,viele schnell durcheinander fahrende Linien [könnten, R. R.] den Zorn [...] abbilden. "52 Drittens ist die Verbindung von Wolke und gezackter Linie (Blitz) eine direkte Anleihe bei Darstellungen Jupiters, des zornigen Gottes, der, in Wolken thronend, Blitze schleudert (Abb. 3). Sinnett und Leadbeater hatten bereits aus älteren Farbenlehren geschöpft, Annie Besant baut einerseits auf deren Aufsätzen auf, macht andererseits weitere Anleihen bei Kunst und Kunstdiskursen. Das ist umso weniger überraschend, als sie in London, bevor sie sich der Theosophie zuwandte, in künstlerisch-intellektuellen Kreisen verkehrte. Sie war mit dem Dichter George Bernard Shaw eng befreundet und stand in Verbindung mit dem Künstler William Morris. ${ }^{53}$

Zwar gibt es in den ersten Jahren nach Blavatskys Tod noch weitere Publikationen über diesen Themenbereich, ${ }^{54}$ soweit ich das beurteilen kann, wurde der theosophische Diskurs im Wesentlichen jedoch durch die drei Aufsätze von Sinnett, Leadbeater und Besant geprägt. Nach der Veröffentlichung dieser Texte war die Materie so wichtig und umfangreich geworden, dass ihre Abhandlung in Buchform weiter geführt wurde. Ein erster theosophischer Band zu diesem Thema, vermutlich das älteste Buch über die Aura überhaupt, erscheint bereits im Dezember 1896. Der Verfasser, Auguste Jean Baptiste Marques (1841-1929), ist Gründungspräsident der Hawaiischen Theosophischen Gesellschaft. Anders als Sinnett, Leadbeater und Besant gehört er nicht zur ersten Reihe des theosophischen Führungszirkels. Er beruft sich ausführlich auf Sinnetts Aufsatz und übernimmt dessen knappen Titel, The Human Aura. ${ }^{55}$ Obgleich er sich kritisch über Leadbeater und Besant äußert, paraphrasiert er ihre Texte und Bilder. So ist, um bei unserem Beispiel zu bleiben, seine Darstellung von ,physical Fear with anger, a black and grey mist, with electrical flashes of explosive passion“ (Fig. 7 in Abb. 3) eine Zusammenstellung des Blitzes aus der Wolke, wie es Besant für „brutal anger“ gezeichnet hatte, mit ihrer zentrifugalen Darstellung des „explosive anger“ (Fig. 4 und 6 in Abb. 2).

Retrospektiv betrachtet kann man diese Publikationen der 1890er Jahre als Auftakt für die im ersten Jahrzehnt des 20. Jahrhunderts erschienenen und bereits

52 Moses Mendelssohn: Über die Empfindung (1755). Gesammelte Schriften. Jubiläumsausgabe. Hg. v. Fritz Bamberger. Bd. 1: Schriften zur Philosophie und Ästhetik I. Berlin 1929, S. 116. Zur Geschichte des ästhetischen Diskurses über Linien siehe Rosenberg: Abstraktion (wie Anm. 7), S. 19-32.

53 Taylor: Besant (wie Anm. 35), passim.

54 Siehe die Bibliographie von Auguste Jean Baptiste Marques: The Human Aura. San Francisco 1896, S. 75f.

55 Das Copyright ist auf Dezember 1896 datiert. Besants Aufsatz von 1896 wird kritisch rezipiert (ebd., S. 36), aber im Gegensatz zu Leadbeaters Text von 1895 nicht in Marques Literaturverzeichnis aufgenommen. Umgekehrt ist eine kritische Rezension von Marques Buch in Lucifer 19 (Feb. 1897), S. 522f., signiert A.M.G., erschienen. 
erwähnten theosophischen Klassiker bezeichnen: Man Visible and Invisible (1902 von Leadbeater, Abb. 5) und Thought Forms (1905 von Besant und Leadbeater, Abb. 11). Zwei komplementäre Bände, die eine systematische Kartographie der Aura konstruieren, einen Atlas des Übersinnlichen im Mikrokosmos. Während die Schriften der 1890er Jahre nur von einem kleinen theosophischen Kreis gelesen und entsprechend von der neueren Forschung übersehen wurden, sind die Bücher von 1902 und 1905 mehrfach aufgelegt und vielfach übersetzt worden. Seit Sixten Ringbom stehen sie im Mittelpunkt der kunsthistorischen Auseinandersetzung mit Esoterik. Im ersten, Man Visible and Invisible, führt Leadbeater die Überlegungen aus, die er im Aufsatz über die Aura sieben Jahre zuvor niedergelegt hatte. Dies wird bereits im Frontispiz deutlich, wo er die Farbskala des Aufsatzes fast wörtlich wiedergibt, allerdings mit visuellen Mitteln (Abb. 5). ${ }^{56} \mathrm{Zu}$ sehen ist eine Palette aus 25 quadratischen Farbproben, wie sie im 19. Jahrhundert sowohl in Kunstlehrbüchern (Abb. 6), ${ }^{57}$ als auch in Kunsthandlungen üblich waren. ${ }^{58}$ Leadbeater geht es allerdings nicht um die Anordnung, Herstellung und/oder Benennung der einzelnen Farbtöne, sondern um ihre Bedeutung im Hinblick auf die seelische und geistige Verfassung desjenigen, in dessen Aura sie vorkommen. Das helle Violett links oben ist beispielsweise Ausdruck von ,High Spirituality“, das Rosa in der Mitte von „High Unselfish Affection“, das Schwarz rechts unten von ,Malice“. Das Buch enthält 25 weitere Tafeln, die als farbige Offsets gedruckt wurden. ${ }^{59}$ Die meisten (22) präsentieren verschiedene Formen der menschlichen Aura - ovale Bilder, in denen wolkige Farben und Muster komponiert sind. Die Vielfalt dieser Bilder und die Qualität der Entwürfe sind bemerkenswert. Vier Beispiele mögen das veranschaulichen. Zuerst erläutert Leadbeater die Aura eines ,Savage', der mit wulstigen Lippen und breiter Nase negroide Züge trägt (Abb. 7). Allen Beteuerungen der Theosophie über die Gleichheit der Menschen zum Trotz ist die koloniale Einstellung des Autors unübersehbar. Leadbeater führt aus, dass das Bild das ungezügelte, ganz von der Sinnlichkeit bestimmte Seelenleben des Wilden widerspiegelt. ${ }^{60}$ Die ungeordneten, form- und symmetrielosen Farbflächen werden als

56 Vgl. mit Leadbeater: The Aura (wie Anm. 38), S. 139 wo es beispielsweise heißt: „Thick, black clouds in the aura usually indicate hatred and malice. [...] Luminous lilac-blue, which indicates higher spirituality“. Auch die Reihenfolge der Farben ist beibehalten worden, von unten rechts nach oben links verlaufend. Die Beschreibung der Farben wird im Buch in einem eigenen Kapitel noch einmal etwas ausführlicher diskutiert (Leadbeater: Man Visible [wie Anm. 5], S. 80-86).

57 Siehe beispielsweise auch M[ary] Gartside: An Essay on Light and Shade, on Colours, and on Composition in General. London 1805, Tab. I u. II.

58 Etwa von der Wasserfarbenfirma Winsor \& Newton (siehe ein 1898 datiertes Beispiel unter URL: http://www.etsy.com/listing/78120621/watercolor-paint-chart-sale-vintage [30.10.2011]).

59 Sie werden, eigentlich irreführend, als „Photochromogravuren“ bezeichnet und sind von Lyons \& London hergestellt.

60, ,[I]n turning to the corresponding astral body (Plate VII.) we shall find it almost entirely uncontrolled. Accordingly, we see how enormous a proportion of this vehicle of desire is occu- 
Entsprechungen eines ungeschliffenen Innenlebens verstanden. Die Analogie ist vergleichbar mit Besants bereits zitierter Aussage, wonach die Klarheit der Umrisse der Bestimmtheit von Gedanken entspreche. Scharf umrissene rote Blitze, die aus schwarzen Wolken hervorspringen, sind das Hauptmotiv der Aura eines heftig erzürnten Mannes (Abb. 8), eine Zeichnung, die eine weitere Adaptation von Besants Bild des „brutal Anger“ (Fig. 4 in Abb. 2) ist. Abbildung 9 stellt die Aura eines reizbaren Menschen dar (,irritable man“). Die scharlachroten Striche, die wie Verletzungen von oben auf ihn herabprasseln, beschreibt Leadbeater als Ausrufezeichen und deutet sie als die vielen Verärgerungen, durch die sich der Mann aus der Ruhe bringen lässt. ${ }^{61}$ Schließlich ist die Aura einer deprimierten Person (Abb. 10, „deep depression“) durch parallel verlaufende graue Ringe bestimmt. Der als Silhouette etwas undeutlich auszumachende deprimierte Mann scheint in einem nebelartigen Käfig eingesperrt zu sein, aus dem er nicht auszubrechen weiß - Leadbeater spricht an dieser Stelle mit poetischer Verve vom „Nebel der Verzweiflung".62 Auch hier wird deutlich, dass die Bilder der Aura in der wirkungsästhetischen Tradition wurzeln, da beispielsweise bereits Lomazzo die Farbe Grau mit Trauer und Melancholie in Verbindung gebracht hatte. ${ }^{63}$ Leadbeaters Rückgriffe auf die Kunstgeschichte reichen aber noch weiter. Wenn er einen „durchschnittlichen“ bzw. „fortgeschrittenen“ Menschen (im Gegensatz zum Wilden) darstellen will, so verwendet er die Silhouette von Polyklets Doryphoros (Abb. 9), jener Skulptur, die im Griechenland des 5. Jahrhunderts den Proportionskanon des Menschen veranschaulichen sollte. ${ }^{64}$ Leadbeater gibt an, dass der Theosoph Graf Maurice Prozor die Bilder nach den Angaben des Autors gemalt habe. ${ }^{65}$ Prozor könnte mit dem gleichnamigen, um 1900 aktiven Übersetzer von Ibsens Dramen ins Französische identisch sein. Als bildender Künstler lässt er sich aber

pied exclusively by sensuality, indicated by the very unpleasant brown-red which is almost blood-colour" (Leadbeater: Man Visible [wie Anm. 5], S. 88).

61 „The case of the irritable man [...] what especially differentiates him [...] is the presence in all parts of the astral body of little floating flecks of scarlet, somewhat like notes of exclamation. These are the result of little accessions of irritation at the small worries which are constantly occurring in ordinary life. [...] These spots gradually fade away, but their places are taken by others, for the irritable man is never at a loss for subjects of annoyance" (ebd., S. 109).

62 ,[T]here are only too many who yield themselves to these feelings, and allow the fog of despair to close round them until all the world looks black" (ebd., S. 111).

63 „Or per cominciare, troviamo che i colori neri, lucidi, terrei, plumbei, \& oscuri generano per gli occhi nell'animo, riguardante della qualità loro laquale non è altro che tristezza, tardità, pensiero, melancolia, \& simili“" (Lomazzo: Trattato [wie Anm. 49], S. 201). Weitere Vergleichsstellen aus der neueren Literaturgeschichte in Günter Butzer: Metzler-Lexikon literarischer Symbole. Stuttgart u.a. 2008, S. 137f.

64 Leadbeater verwendet neben dem „Savage“ (Taf. V-VII) sowohl die Silhouette des Doryphoros mit angewinkeltem linken Arm (für die namengebende Lanze, die er ursprünglich trug, Taf. VIII-X, XIV-XV, XVII-XVIII, XXV), wie auch eine Variation davon, bei der der rechte Arm in die Hüfte gestemmt ist (Taf. XI-XII, XVI, XIX-XXIV). Die nur schwer auszumachende Silhouette der Taf. XIII ist vermutlich eine dritte Variante.

65 Leadbeater: Man Visible (wie Anm. 5), Author's Note auf der ersten unpag. Seite. 
nicht nachweisen. Die Frage, wie groß sein Anteil an den Kompositionen war, muss vorerst offen bleiben, ${ }^{66}$ man kann und muss aber festhalten, dass die Bilderfindungen sehr originell sind und dass es ihnen auf bemerkenswerte Weise gelingt, Stimmungen und Eigenschaften sichtbar zu machen. Es sind gelungene (Kunst) werke, obgleich sie, zumindest explizit, keinen ästhetischen Absichten folgen.

Leadbeaters Buch ist inhaltlich nichts anderes als eine ausdifferenzierte und um viele Details bereicherte Durchführung seines älteren Aufsatzes. Die entscheidende Neuerung besteht aber darin, dass er dem Beispiel Besants folgt und hier mit Bildern arbeitet. Damit entsteht eine neuartige Qualität, die maßgeblich zum Erfolg der Publikation beigetragen haben dürfte. Die gesamte Argumentation des Buches beruht auf der Wechselwirkung zwischen Text und Bild - etwa so wie bei kunsthistorischen Publikationen. Der Text erläutert das Bild, und das Bild macht die Aussage, d.h. die Aura, anschaulich und damit plausibel. Das ähnelt der Vorgehensweise von Besants Aufsatz von 1896 (Abb. 2). Im Vergleich dazu sind Leadbeaters Bilder (Abb. 7-10) komplexer und - zumindest graduell - anschaulicher. Sie emanzipieren sich vom Status der Illustration. Anders als bei Besant ist der Text weder in der Lage noch willens, die Bilder vollständig zu erklären, ihre Bedeutung gänzlich zu übersetzen. Wichtiger als die Erklärung ist, dass die Bilder eine hohe Evidenz besitzen, ihre Bedeutung leuchtet in der Regel ein. Leadbeater überzeugt einerseits als Schriftsteller durch eine anschauliche Beschreibung des Bildes und andererseits als Maler durch gelungene Charakterisierungen. Er erreicht diese Evidenz dadurch, dass er die in Kunsttheorien diskutierten Eigenschaften von Farbtönen und Linien verwendet und auf tradierte ikonographische Elemente zurückgreift. Ob derartige Zuordnungen - Grau für Trauer, rote Blitze für Zorn kulturell verschieden, dem historischen Wandel unterliegen oder aber evolutionär bedingt und damit anthropologisch konstant sind, braucht an dieser Stelle nicht diskutiert zu werden.

Die Kartographie der Aura war der Mitte der 1890er Jahre ein gemeinsames Projekt von Annie Besant und Charles Leadbeater. Wenn Besant 1896 von „two clairvoyant Theosophists“ als Urheber der Erkenntnisse über die Aura spricht, so kann kein Zweifel daran bestehen, dass Leadbeater der zweite war. Ihre Zusammenarbeit auf diesem Gebiet wird allerdings erst 1905 aktenkundig, als sie das Buch Thought-Forms gemeinsam veröffentlichen. Das Werk hat das gleiche Format und ein fast identisches Frontispiz (Abb. 11) wie Man Visible and Invisible (Abb. 5). Während Letzteres die Aura des Menschen erläutert, wird nun behauptet, dass Gefühle, Gedanken und Musik eigene übersinnliche Erscheinungen besitzen, die als Gedankenformen (thought-forms) bezeichnet und detailliert vorgestellt werden. Es handelt sich um eine stark überarbeitete und im Text und Bildteil erweiterte Form des gleichnamigen Aufsatzes Besants von 1896. Von Grund auf neu

66 Skizzen und weitere Unterlagen zu dieser Publikation könnten in dem derzeit schwer zugänglichen Archiv der Theosophischen Gesellschaft in Adyar erhalten sein. 
ist das letzte Kapitel, Forms Built by Music, ${ }^{67}$ das drei bemerkenswerte Bilder von Konzerten beinhaltet (Abb. 12). Bilder, die der Hellseher angeblich noch Stunden nach der Aufführung über dem Aufführungsort - einer kleinen Kirche mitten in der Landschaft - wahrnehmen kann. ${ }^{68}$ Bei der thought-form des hier abgebildeten Konzertes von Richard Wagner sollen die überaus monumentalen, bergförmigen Gebilde den Gesamteindruck der Musik wiedergeben:

[F]or no other composer has yet built sound edifices with such power and decision. [...] in Plate W[agner] we have a still greater and richer form, in the depiction of which all detail is avoided, in order that the full effect of the piece as a whole may be approximately given. ${ }^{69}$

Die Idee eines musikalischen Bildes, das sichtbar und simultan ganze Konzerte in zweidimensionale farbige Flächen umsetzt, war damals nicht neu. Sie lässt sich bis 1725 zurückverfolgen und geht, wie ich andernorts ausführlich gezeigt habe, auf Louis-Bertrand Castel (1688-1757), einen der frühen Verfechter einer wirkungsästhetischen Farbenlehre, zurück. ${ }^{70}$ Castel verwendete dabei bereits die auch von Besant und Leadbeater aufgegriffene Vorstellung von Vibrationen als tertium comparationis zwischen Musik- und Farbtönen: Beide verursachen Vibrationen, durch die sie sich dem Rezipienten vermitteln. Die Äquivalenz bestimmter musikalischer und farblicher Töne erklärt sich durch die Gleichheit ihrer Vibrationen. Besants und Leadbeaters Rezeption der Castelschen „Farbenmusik“ zeigt einerseits, wie tief ihre Auseinandersetzung mit der wirkungsästhetischen Tradition ist, andererseits wie sehr sie sich für Kunst - in diesem Fall Musik - interessieren. Castel hatte 1725 die Umsetzung von Konzerten in ungegenständliche Bilder als harmonische Wandbehänge (tapisseries harmoniques) bezeichnet. Er vergleicht sie mit marmoriertem Papier und erklärt, dass jeder Ton, jede Harmonie, jeder Rhythmus umzusetzen sei. Er selbst hat allerdings nie versucht, solche Wandbehänge zu realisieren. Abstrakte Bilder nach musikalischen Stücken werden erst im 20. Jahrhundert gemalt. ${ }^{71}$ Besant und Leadbeater sind mit Mikalojus Čiurlionis (1875-1911) die ersten, die solche Übersetzungen verwirklicht haben.

Mit den Musikbildern in Thought-Forms (1905) schließt sich nach zwei Jahrzehnten der Kreis, der 1886 mit Blavatskys Interesse für das Sehen von Farben beim Hören von Musik (Synästhesie) begonnen hat. Er führte zu einer esoterischen Re-

67 Besant, Leadbeater: Thought-Forms (wie Anm. 5), S. 75-84.

68 „Such forms remain as coherent erections for some considerable time - an hour or two at least; and during all that time they are radiating forth their characteristic vibrations in every direction" (ebd., S. 78).

69 Ebd., S. 82f.

70 Siehe Rosenberg: Abstraktion (wie Anm. 7), S. 44-46 u. 307-309.

71 Vom Klang der Bilder. Die Musik in der Kunst des 20. Jahrhunderts. Hg. v. Karin von Maur. München 1985; Judith Zilczer: „Color Music“: Synaesthesia and Nineteenth-Century Sources for Abstract Art. In: Artibus et Historiae 8/16 (1987), S. 101-126; Sons \& lumières. Une histoire du son dans l'art du XXe siècle. Hg. v. Sophie Duplaix. Paris 2004; Andrea Gottdang: Vorbild Musik. Die Geschichte einer Idee in der Malerei im deutschsprachigen Raum 17801915. München, Berlin 2004. 
zeption von Kunst und Kunsttheorien und zu originellen Bildern. An dieser Stelle ist es wichtig, den Unterschied zwischen Synästhesie auf der einen Seite und der Beschreibung von Eigenschaften in der Tradition der wirkungsästhetischen Theorie andererseits zu unterstreichen. ${ }^{72}$ Synästhesien stellen sich bei denjenigen, die davon betroffen sind, automatisch und zwangsläufig mit jeder entsprechenden Sinneswahrnehmung ein. Sie können nicht unterdrückt werden, und sie sind in hohem Maße individuell verschieden. Welche Bereiche miteinander verknüpft und vor allem wie diese verknüpft werden, ist bei jedem Synästhetiker anders. Nehmen wir beispielsweise die häufigen synästhetischen Verknüpfungen von Vokalen und Farben: I ist für den einen Weiß, für den anderen Karminrot und für den dritten Blau. ${ }^{73}$ Die wirkungsästhetische Tradition erhebt demgegenüber den Anspruch, objektive Effekte oder zumindest intersubjektiv nachvollziehbare Qualitäten zu beschreiben - etwa, dass Rot eine aktive, Blau dagegen eine passive Farbe sei. Gerade dieses universalistische Postulat macht die wirkungsästhetische Theorie für die monistische Esoterik attraktiv.

Zusammenfassend lässt sich festhalten, dass es im späten 19. und frühen 20. Jahrhundert einen fruchtbaren Austausch zwischen Kunst und Esoterik gegeben hat. Künstler waren insbesondere an der Theosophie interessiert und fanden dort, so wie viele andere Zeitgenossen, Alternativen zu Positivismus und Materialismus auf der einen und zu den tradierten Kirchen auf der anderen Seite. Viele Maler wurden durch okkultistische Vorträge und Schriften inspiriert, etwa bei den Themen ihrer Kunst. ${ }^{74}$ Umgekehrt schöpften Annie Besant und Charles Leadbeater bei der Konstruktion der ,Aura' entscheidende Anregungen aus Kunstgeschichte und Kunsttheorie. Im Hinblick auf die ,Erfindung ' der Abstrakten Kunst muss damit der These von Sixten Ringbom widersprochen werden. Die Tatsache, dass Kandinsky sich für die Theosophie, kaum aber für die Publikationen von Besant und Leadbeater interessiert, lässt sich leicht erklären: Was er dort über die Bedeutung von Farben und Formen fand, war ihm weitgehend bekannt. Die wirkungsästhetischen Grundlagen der Abstrakten Kunst, deren wichtigste Wurzeln im 18. Jahrhundert liegen, haben die Okkultisten von den Künstlern und Kunsttheoretikern gelernt - und nicht umgekehrt.

72 Selbst in der einschlägigen Literatur wird diese Unterscheidung häufig übersehen, etwa bei Mahling. Siehe beispielsweise seine Ausführungen über Kandinsky (Mahling: Audition colorée [wie Anm. 17], S. 257).

73 Siehe die sehr aufschlussreiche Zusammenstellung verschiedener früher Forscher und Dichter in Schrader: Sinnesverknüpfungen (wie Anm. 11), S. 37.

74 Siehe zwei exemplarische Studien, die jeweils für Österreich und die Niederlande breitgefächerte Untersuchungen geleistet haben: Astrid Kury: „Heiligenscheine eines elektrischen Jahrhundertendes sehen anders aus [...]". Okkultismus und die Kunst der Wiener Moderne. Wien 2000, und Marty Bax: Het web der schepping theosofie en kunst in Nederland van Lauweriks tot Mondriaan. Amsterdam 2006. 


\section{Steiner: Wirkungsästhetische Erfahrungen als Vorbedingung und Kompensation für übersinnliche Erlebnisse}

Die Konstruktion der Aura erreicht mit den Büchern Besants und Leadbeaters von 1902 und 1905 einen Höhepunkt und zugleich einen Abschluss. Mir sind keine späteren theosophischen Publikationen bekannt, die das Thema in größerem Umfang weitergeführt hätten. Die Einbindung von Kunst und Kunsttheorie in die Esoterik ist aber vor allem von Rudolf Steiner vertieft und verstärkt worden. Er hat die theosophische Rezeption wirkungsästhetischer Theorien weit über die von Besant und Leadbeater gesetzten Maßstäbe hinaus geführt und im Rahmen seiner Anthroposophie den Künsten eine zentrale Stellung eingeräumt.

Der österreichische Gelehrte, Feuilletonist und Vortragsredner Rudolf Steiner (1861-1925) kommt im Herbst 1900 in Kontakt mit theosophischen Kreisen in Berlin. Der Freidenker wendet sich damals der Esoterik zu. Bereits im Oktober 1902 erfolgt seine Wahl zum Sekretär der neu gegründeten deutschen Sektion der Theosophischen Gesellschaft. Binnen weniger Jahre wird er zum populärsten Okkultisten des kontinentalen Europa. ${ }^{75}$ Die Aura ist neben der Geschichte von Mysterien und Mystik sowie der Seelenwanderung eines der ersten theosophischen Themen, mit denen sich Steiner intensiv beschäftigt. Im Juni 1903 begründet er in Anlehnung an das gleichnamige von Annie Besant herausgegebene Publikationsorgan eine deutsche theosophische Zeitschrift namens Luzifer $^{76}{ }^{7 m}$ vierten Heft dieser Zeitschrift (September 1903) setzt er sich bereits mit Leadbeaters Bildern der Aura auseinander. Anfang 1904 verfasst er eine einschlägige Artikelserie Von der Aura des Menschen. ${ }^{77}$ Der Vergleich mit den entsprechenden Schriften von Leadbeater und Besant ist aufschlussreich, auch um Steiners Position am Anfang seiner okkultistischen Laufbahn zu bestimmen. Als erstes fällt der unterschiedliche Sprachduktus auf: Steiners Argumentation ist zwar kolloquialer vorgetragen, ${ }^{78}$ intellektuell aber weitaus anspruchsvoller, erkenntnistheoretisch reflektierter. Darüber hinaus sind Steiners Aussagen komplexer und vielschichtiger. Deutlicher als

75 Zur Biographie Steiners siehe zuletzt Zander: Steiner (wie Anm. 46).

76 Seit 1904 Lucifer mit der Gnosis bzw. Lucifer Gnosis.

77 Rudolf Steiner: Die Kultur der Gegenwart im Spiegel der Theosophie. Bruno Wille und C. W. Leadbeater. In: Luzifer. Zeitschrift für Seelenleben und Geisteskultur 1 (1903), S. 161-167 und Rudolf Steiner: Von der Aura des Menschen. In: Lucifer mit der Gnosis 2 (1904), S. 3-7, 4145, 73-76 u. 105-107.

78 Dies liegt vermutlich daran, dass der Text als Vortragsreihe entstand. Hans Schmidt: Das Vortragswerk Rudolf Steiners. Dornach 1950 nennt zwar keinen derartigen Titel in diesem Zeitraum, im Rudolf-Steiner-Archiv lassen sich aber mindestens zwei Vorträge über die Aura aus dem Jahr 1904 nachweisen, einer davon mit Datum 12.01.1904 (Hinweis von Roland Halfen). Auf die zentrale Rolle der Mündlichkeit in Steiners Werk verweist Peter Sloterdijk in einer Podiumsdiskussion im Vitra Design Museum am 14.10.2011 (Mitschnitt über YouTube zugänglich). Vgl. Ulrich Kaiser: ,Wann wird das symbolische Gewand fallen?` Dogma und Methode. Zur Hermeneutik des Steinerschen Werks. In: Die Drei 8-9 (2011), S. 41-55, hier S. 55. 
Besant und Leadbeater betont er die Differenz zwischen der übersinnlichen Schau und der visuellen Wahrnehmung ${ }^{79}$ und vermeidet sowohl Illustrationen wie auch allzu scharfe Festlegungen der Bedeutung einzelner Farben. Ein deutlicher Unterschied besteht schließlich im Umgang mit fremden Texten. Leadbeater verweist gerne auf eigene Schriften, gelegentlich auch auf andere Theosophen, verwendet aber kaum direkte Zitate. Steiner zitiert dagegen viel und ausführlich, insbesondere Dichter (Goethe und Hamerling) und Naturwissenschaftler (Tyndall und Du BoisReymond). ${ }^{80}$ Damit schlägt er eine Brücke zum bildungsbürgerlichen zeitgenössischen Diskurs, an dem er kurz zuvor noch sehr aktiv teilgenommen hatte.

Die Unterschiede zwischen Steiner auf der einen, Besant und Leadbeater auf der anderen Seite sind Ausdruck verschiedener Denk- und Schreibstile, inhaltlich überwiegen aber die Gemeinsamkeiten. Obwohl sich Steiner von Leadbeater explizit distanziert und sich auf eigene übersinnliche Erfahrungen beruft, ${ }^{81}$ ist nicht zu übersehen, dass seine Erkenntnisse im Wesentlichen in einer theosophischen Bibliothek gewonnen wurden, besonders bei der Lektüre von Leadbeaters Man Visible and Invisible. Steiner übernimmt das neue theosophische Konzept der Aura als Bild des Innenlebens des Menschen, in dem „Farbenwellen [...] nicht nur die Grundverfassung der Seele, sondern auch vorübergehende Affekte, Stimmungen und andere innere Erlebnisse“ zeigen. ${ }^{82}$ Steiner übernimmt auch die Deutung einzelner Farben. In Anlehnung an die oben besprochenen Beispiele (Abb. 8 und 9) schreibt er etwa: „Ein plötzlich ausbrechender heftiger Ärger erzeugt rote Wellen [...] Personen, die durch jeden äußeren Eindruck stark erregt werden, zeigen ein fortwährendes Aufflackern kleiner rötlicher Punkte und Fleckchen in der Aura." ${ }^{83}$ Leadbeater bezeichnet Hellblau als „devotion to a noble ideal“ (Abb. 5). Steiner übersetzt und führt aus: „,ie blauen Farbentöne treten bei hingebungsvollen Natu-

79 „Will sich aber ein Beobachter der übersinnlichen Gebiete verständlich machen, dann muß er in Bildern sprechen, die von dem sinnlichen Leben hergenommen sind. Das wird leicht falsch gedeutet. Man glaubt, es sehe im Übersinnlichen wirklich so aus, wie es die aus der Sinnenwelt genommenen Bilder, deren sich der Darsteller bedienen muß, wörtlich ausdrücken“ (Steiner: Kultur [wie Anm. 77], S. 165).

80 Steiner: Aura (wie Anm. 77), bes. S. 3-5.

81 Steiner rezensiert die deutsche Übersetzung eines Vortrags von Leadbeater (Die Astral-Ebene, ihre Szenerie, ihre Bewohner und ihre Phänomene) und begrüßt diese einerseits als „die übersichtlichste und in gewisser Beziehung beste Schrift über dieses Gebiet". Andererseits äußert er bereits zu diesem Zeitpunkt Zweifel an Leadbeaters Integrität: „Denn - es sei ganz offen gesagt - in diese übersinnlichen Regionen bringt ein jeder seine Vorurteile aus der sinnlichen Welt mit, und diese beeinträchtigen, färben seine Beobachtungen in einer Weise, gegen die unsere Täuschungen in der sinnlichen Welt ganz geringfügig zu nennen sind. [...] Sein [Leadbeaters] Blick ist durchaus nicht unbefangen“ (Steiner: Kultur [wie Anm. 77], S. 166). Bemerkenswert ist, dass Steiner Leadbeaters einschlägiges Buch im eigenen Aufsatz über die Aura nur sehr beiläufig erwähnt (Steiner: Aura [wie Anm. 77], S. 6).

82 Ebd., S. 7. Darüber hinaus teilen Steiner und Leadbeater auch die grundlegende Ansicht, dass der Mensch aus sieben Teilen besteht, von denen nur der unterste physisch sichtbar ist. Diese Vorstellung geht vermutlich auf Blavatsky zurück (ebd., bes. S. 106).

83 Ebd., S. 7. 
ren auf. [...] Je mehr der Mensch sein Selbst in den Dienst einer Sache stellt, desto bedeutender werden die blauen Nuancen." ${ }^{84}$

Mit der Annahme des theosophischen Konzeptes einer farbigen Aura rezipiert Steiner auch die dort eingearbeiteten wirkungsästhetischen Theorien. Im Gegensatz zu seinen theosophischen Vorgängern bezieht er sich allerdings explizit auf einen Vertreter dieser Theorien: auf Goethe. Sein Aufsatz beginnt mit einem langen Zitat der ersten Abschnitte des Vorworts von Goethes Farbenlehre. ${ }^{85}$ Anders als Leadbeater und ähnlich wie Goethe gruppiert Steiner die Farben und ihre Aussagen in polare Gruppen und beginnt mit der Unterscheidung in „braune und braunrote Farbenströmungen“ - die in der Aura von Menschen mit ,stark ausgeprägte[n] Affekte[n]“ zu finden sind - und in ,,verschiedene Nuancen des Grün“, die den „ruhigen, abwägenden, nachdenklichen Menschen“ eigen sind. ${ }^{86}$ Dieser Bezug auf Goethe ist auch vor dem Horizont von Steiners Biographie zu verstehen. Er war von 1884 bis 1897 Herausgeber der naturwissenschaftlichen Schriften Goethes. Er kennt und schätzt die Farbenlehre des Weimarer Dichters und insbesondere dessen Aussagen zur Wirkungsästhetik im Kapitel über die Sinnlich-sittliche Wirkung der Farbe.

Goethe beschreibt - wie Lomazzo, Usher und andere - die ästhetische Wirkung einzelner Farben. Steiner überträgt diese wirkungsästhetischen Beschreibungen wie Leadbeater und Besant - auf die Aura. Steiner geht allerdings weiter als seine theosophischen Vorgänger, indem er auch einen Rückschluss zieht: Er wertet die Wahrnehmung ästhetischer Wirkungen als „,die beste Vorbedingung“ der höheren Erkenntnis auf. Wer mit den Augen des Künstlers die Eigenschaften von Farben, Formen und Linien wahrnimmt, der erfährt bereits etwas, was über das rein Physische hinaus reicht. ${ }^{87}$ Steiner fordert deswegen Wissenschaftler dazu auf, die Welt auch in ihrer ästhetischen Dimension zu betrachten, um ein ganzheitliches Bild des Wesens der Natur zu gewinnen; eine Vorgehensweise, die er „Goetheanismus“ nennt. Das erklärt den enormen Stellenwert der Kunst - von der Malerei, Skulptur

84 Ebd., S. 6. Ausgerechnet an dieser Stelle, bei der Steiners Übernahme besonders deutlich ist, fügt er eine Anmerkung ein, in der er lediglich zum Vergleich mit Leadbeater auffordert: „Ich möchte ausdrücklich bemerken, daß ich mich gerne korrigieren lasse von andern Forschern. Die Beobachtungen auf diesem Felde sind natürlich unsicher. Und diese Unsicherheit läßt sich fast nicht vergleichen mir der, die schon auf dem physischen Felde möglich ist, obwohl doch auch diese - Forscher wissen es - eine sehr große ist. Ich mache zur Vergleichung mit meinen Angaben auf die Schrift C. W. Leadbeaters: ,Man visible and invisible' aufmerksam, die 1902 in London, Theosophical publishing Society, erscheinen ist“" (ebd.).

85 Ebd., S. 3.

86 Ebd., S. 6. Goethe unterscheidet sowohl kalte versus warme wie auch Plus- und Minusseite (siehe Rosenberg: Abstraktion [wie Anm. 7], S. 325, Anm. 132).

87 „Bemerkt soll werden, dass künstlerisches Empfinden, gepaart mit einer stillen, in sich versenkten Natur, die beste Vorbedingung für die Entwicklung der geistigen Fähigkeiten ist. Dieses Empfinden dringt ja durch die Oberfläche der Dinge hindurch und gelangt dadurch zu deren Geheimnissen“ (Rudolf Steiner: Wie erlangt man Erkenntnisse der höheren Welten? In: Lucifer mit der Gnosis II [1904], S. 68). 
und Architektur über Eurythmie bis zum Schauspiel - innerhalb der Anthroposophie. Dieser Rückschluss und die sich daraus ergebende Aufwertung der Kunst sind charakteristisch für die Anthroposophie. Das unterscheidet sie von der Theosophie, von der sie sich abgespalten hat, wie auch von anderen okkultistischen Gruppen, die um 1900 aktiv waren. Anthroposophie ist die einzige dieser Bewegungen, die ein Jahrhundert später weltweit floriert. Ich vermute, dass der wirkungsästhetische Rückschluss entscheidend zu ihrer Nachhaltigkeit beigetragen hat. Der Umgang mit Kunst vermittelt jedem Adepten ästhetische Erfahrungen, die eine Kompensation für die erhofften übersinnlichen Wahrnehmungen sind.

\section{Abbildungsverzeichnis}

Farbabb. 1: Mary Gartside: Scarlet 5.[th Blot], Aquarell, 28x22,3 cm. Aus: Ders.: An Essay on Light and Shade, on Colours, and on Composition in General. London 1805. Privatbesitz.

Farbabb. 2: Annie Besant: Thought-forms: brutal anger (fig. 4), steady anger (fig. 5), explosive anger (fig. 6), Photochromogravure, 24x26,2 cm. Zweite Tafel aus: Ders.: ThoughtForms. In: Lucifer. A Theosophical Magazine XIX (1896), zwischen S. 72 und 73. Privatbesitz.

Farbabb. 3: Jupiter, Stahlstich, 9x13,7 cm. Aus: Illustrirter Kalender für 1850. Jahrbuch der Ereignisse, Bestrebungen und Fortschritte im Völkerleben und im Gebiet der Wissenschaften, Künste und Gewerbe 5 (1850). Berlin, Bibliothek für Bildungsgeschichtliche Forschung (DIPF).

Farbabb. 4: Auguste Jean Baptiste Marques: The Human Aura. San Francisco 1896, S. 37 mit zugehöriger Tafel, 27,5x22,1 cm (Doppelseite). Privatbesitz.

Farbabb. 5: Charles Leadbeater: Man Visible and Invisible. London 1902. Frontispiz, Photochromogravure, 22,4x14,2 cm (Tafel). Privatbesitz.

Farbabb. 6: George Barnard: Table of Greens and Russets, Farbholzstich, 26x18 cm. P1. 13 aus: Ders.: The Theory and Practice of Landscape Painting in Water-Colours. 2. Aufl. London 1861. Privatbesitz.

Farbabb. 7: Maurice Prozor: The Astral Body of the Savage, Photochromogravure, 22,4x14,2 cm. P1. VII aus: Charles Leadbeater: Man Visible and Invisible. London 1902. Privatbesitz.

Farbabb. 8: Maurice Prozor: Intense Anger, Photochromogravure, 22,4x14,2 cm. Pl. XIII aus: Charles Leadbeater: Man Visible and Invisible. London 1902. Privatbesitz.

Farbabb. 9: Maurice Prozor: The Irritable Man, Photochromogravure, 22,4x14,2 cm. Pl. XVI aus: Charles Leadbeater: Man Visible and Invisible. London 1902. Privatbesitz.

Farbabb. 10: Maurice Prozor: Deep Depression, Photochromogravure, 22,4x14,2 cm. P1. XVIII aus: Charles Leadbeater: Man Visible and Invisible. London 1902. Privatbesitz.

Farbabb. 11: Annie Besant und Charles Leadbeater: Thought Forms. London 1905, Frontispiz, Photochromogravure, 24x15,1 cm (Tafel). Privatbesitz.

Farbabb. 12: Musik von Wagner, Photochromogravure, 24x15,1 cm. Aus: Annie Besant und Charles Leadbeater: Thought Forms. London 1905, gegenüber S. 82. Privatbesitz. 


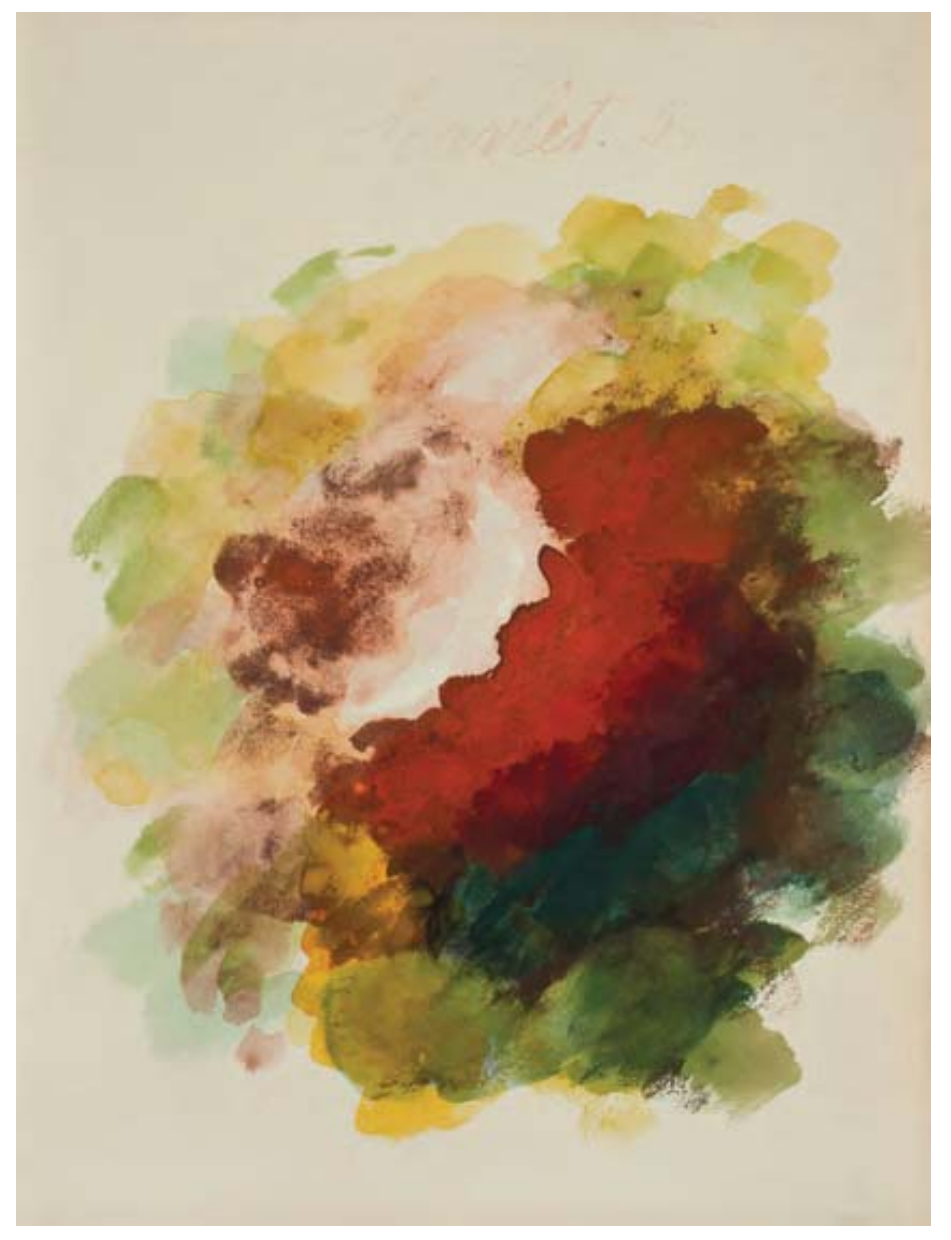

Abb. 1: Mary Gartside: Scarlet 5.[th Blot], 1805

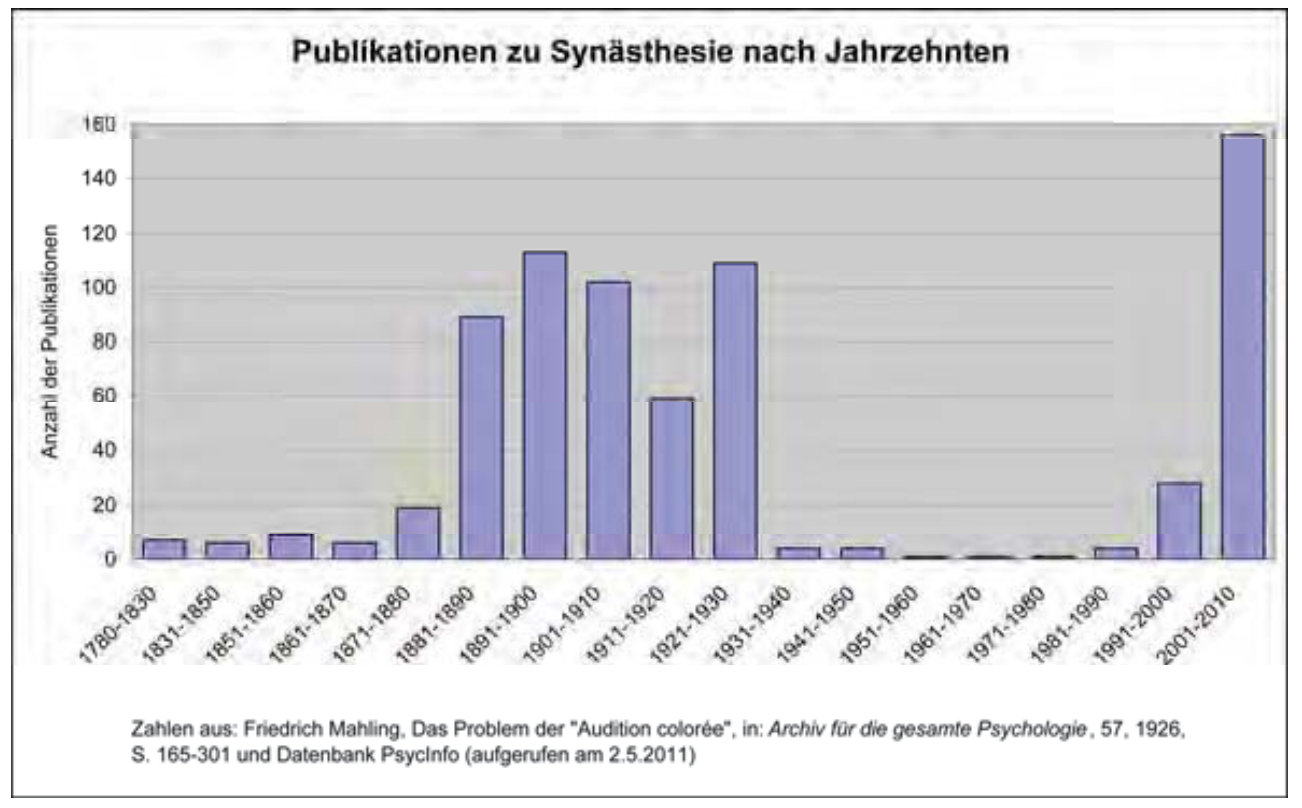

Tab. 1 


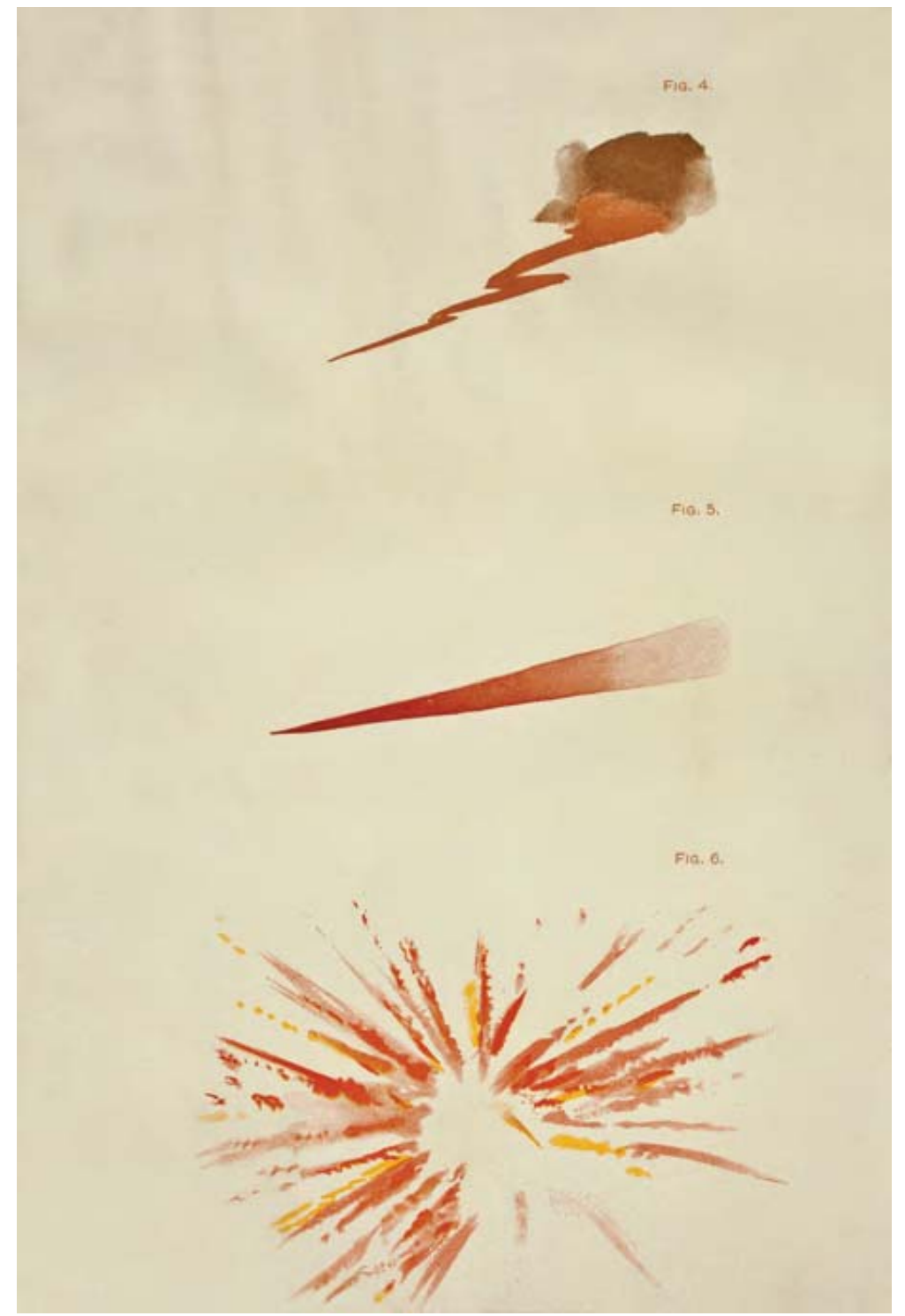

Abb. 2: Annie Besant: brutal anger (fig. 4), steady anger (fig. 5), explosive anger (fig. 6), 1896

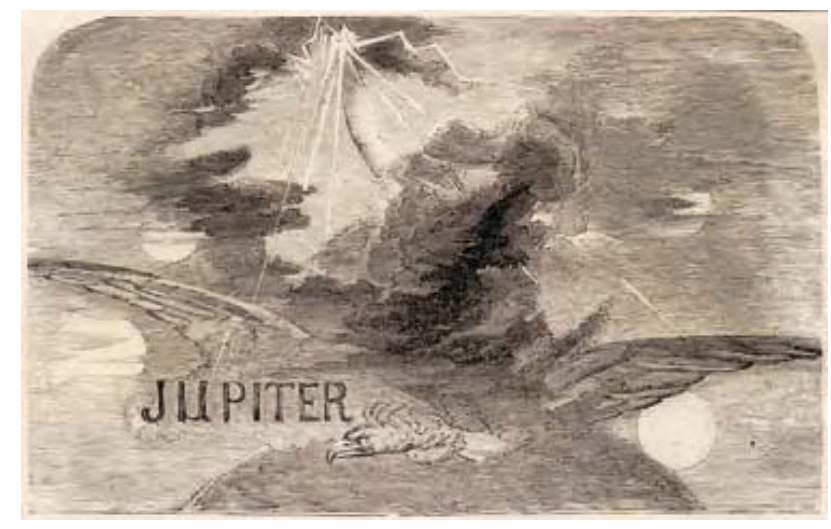

Abb. 3: Jupiter, Stahlstich aus: Illustrirter Kalender für 1850 

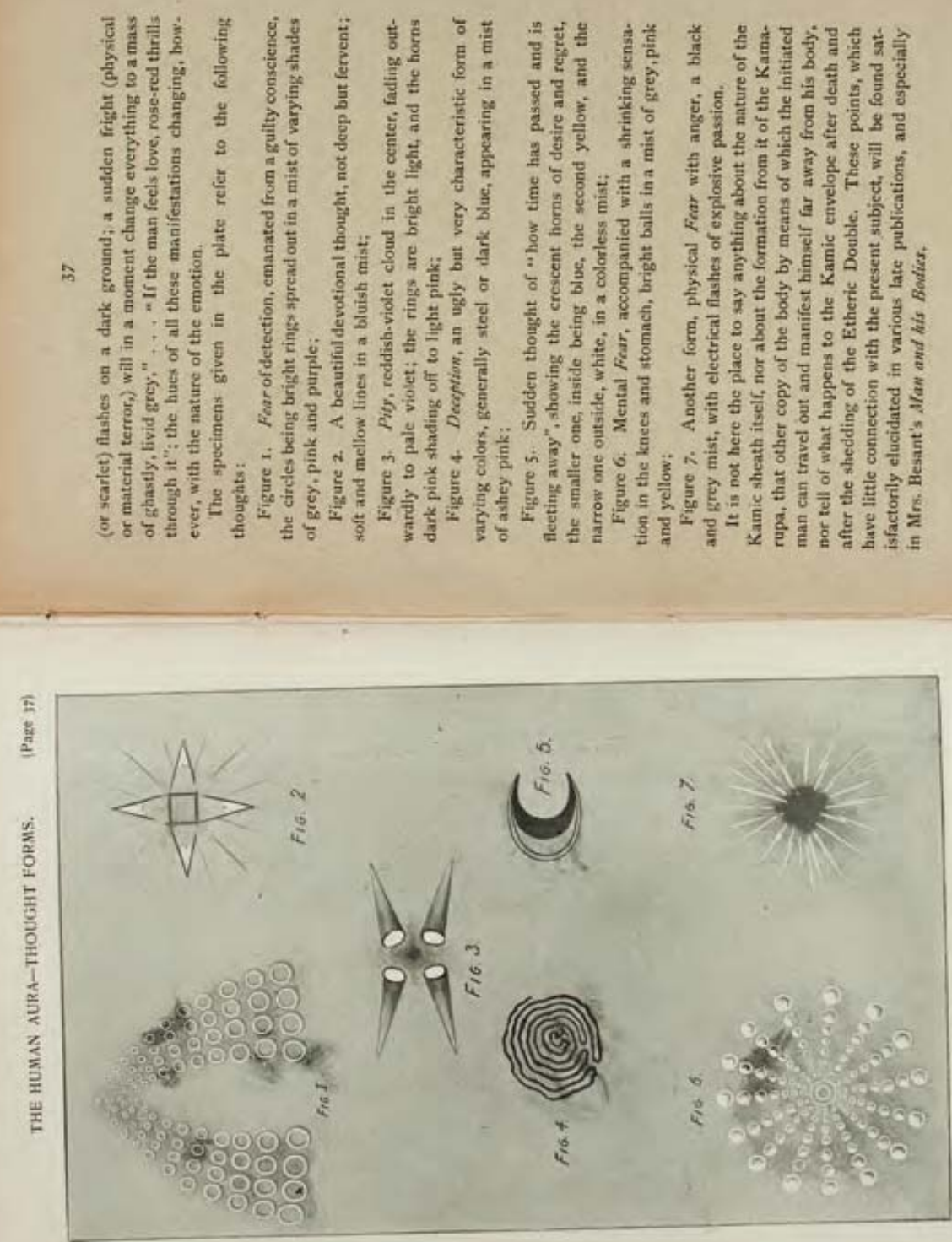

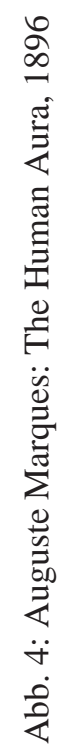




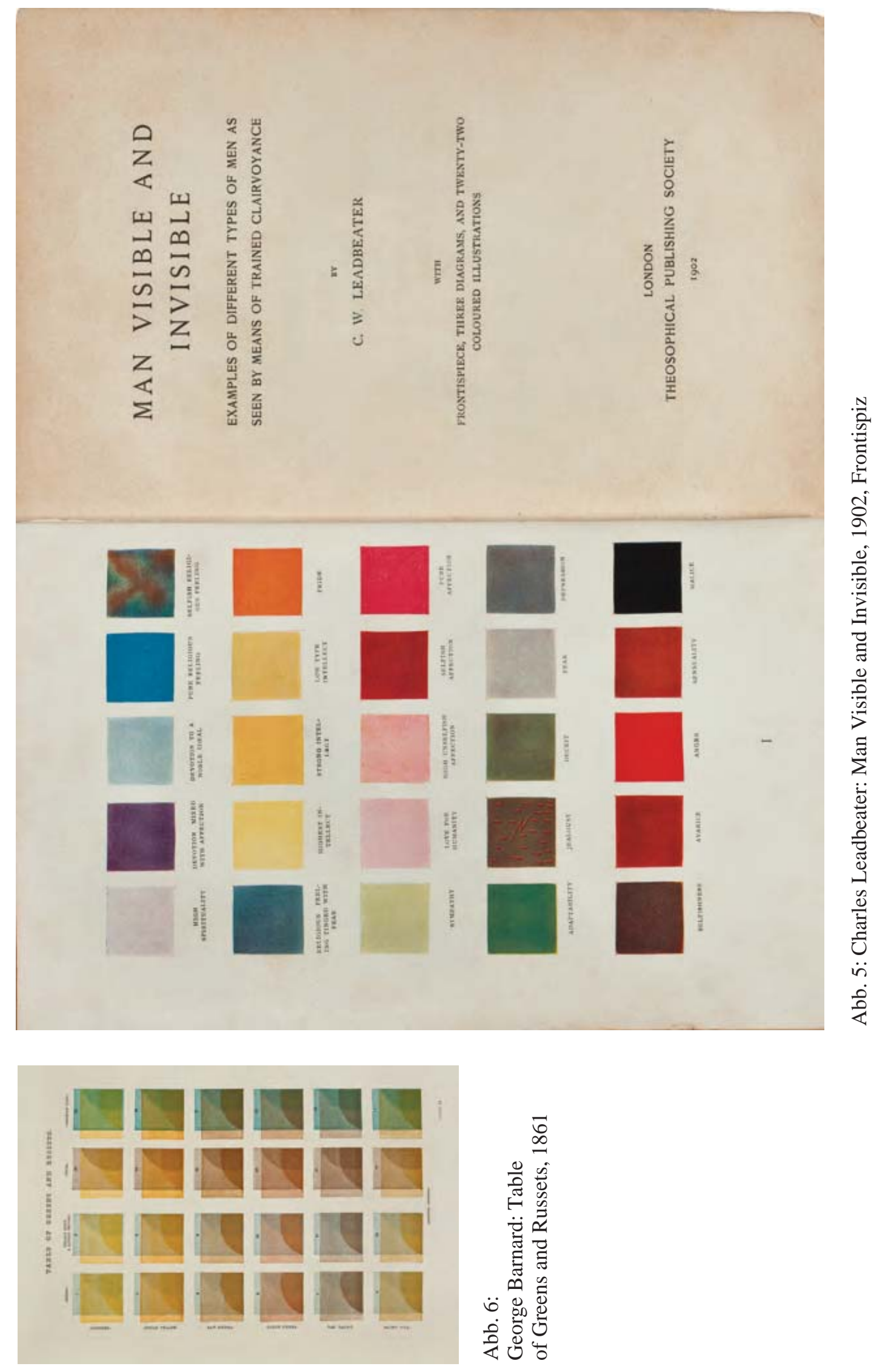




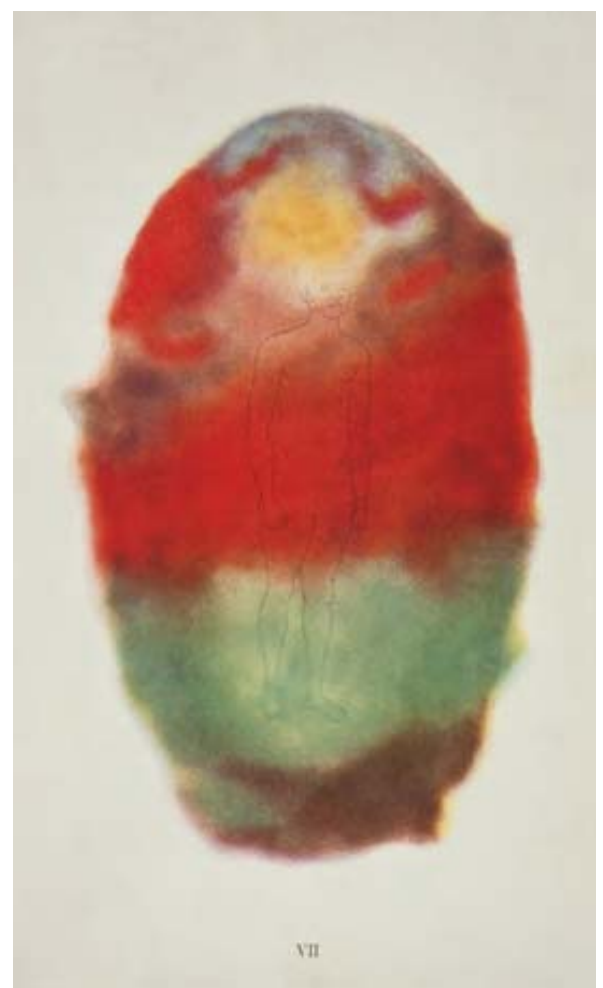

Abb. 7: Maurice Prozor: The Astral Body of the Savage, 1902

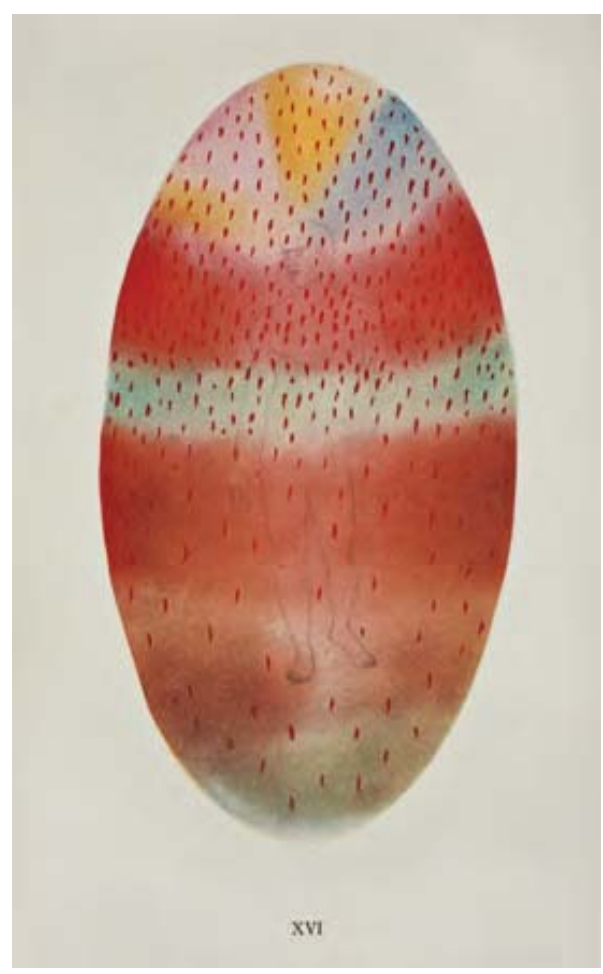

Abb. 9: Maurice Prozor: The Irritable Man 1902

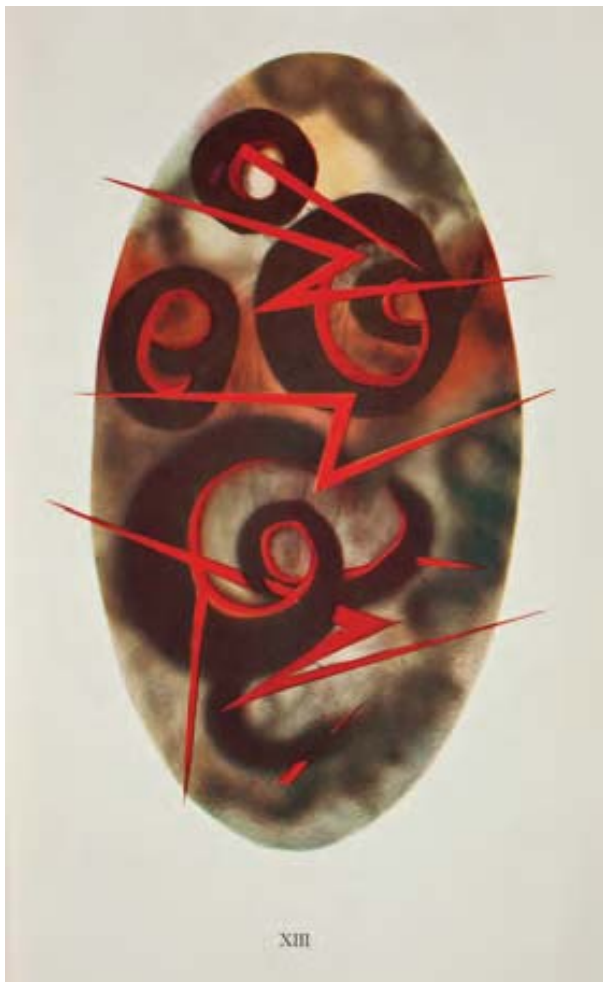

Abb. 8: Maurice Prozor: Intense Anger, 1902

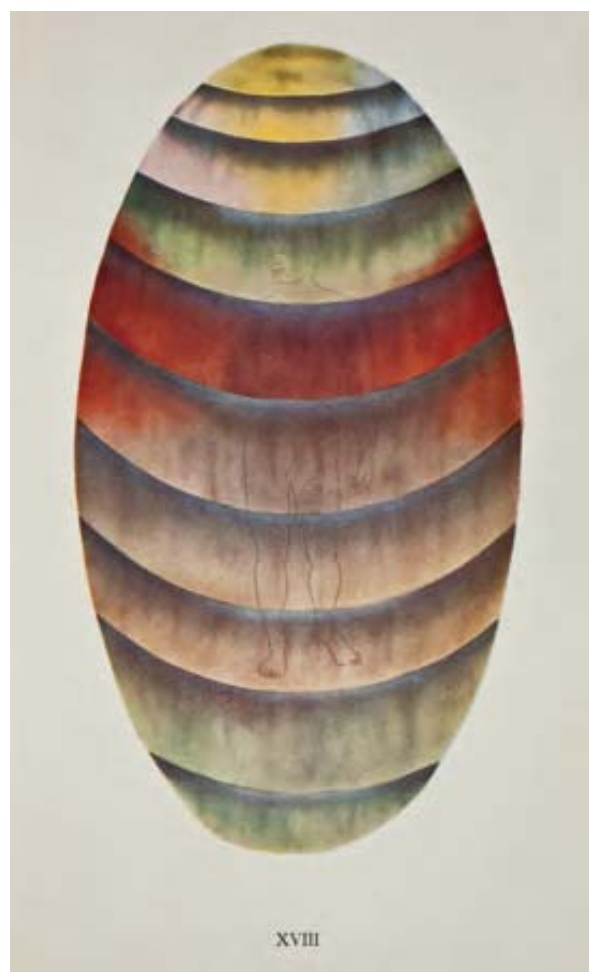

Abb. 10: Maurice Prozor: Deep Depression 


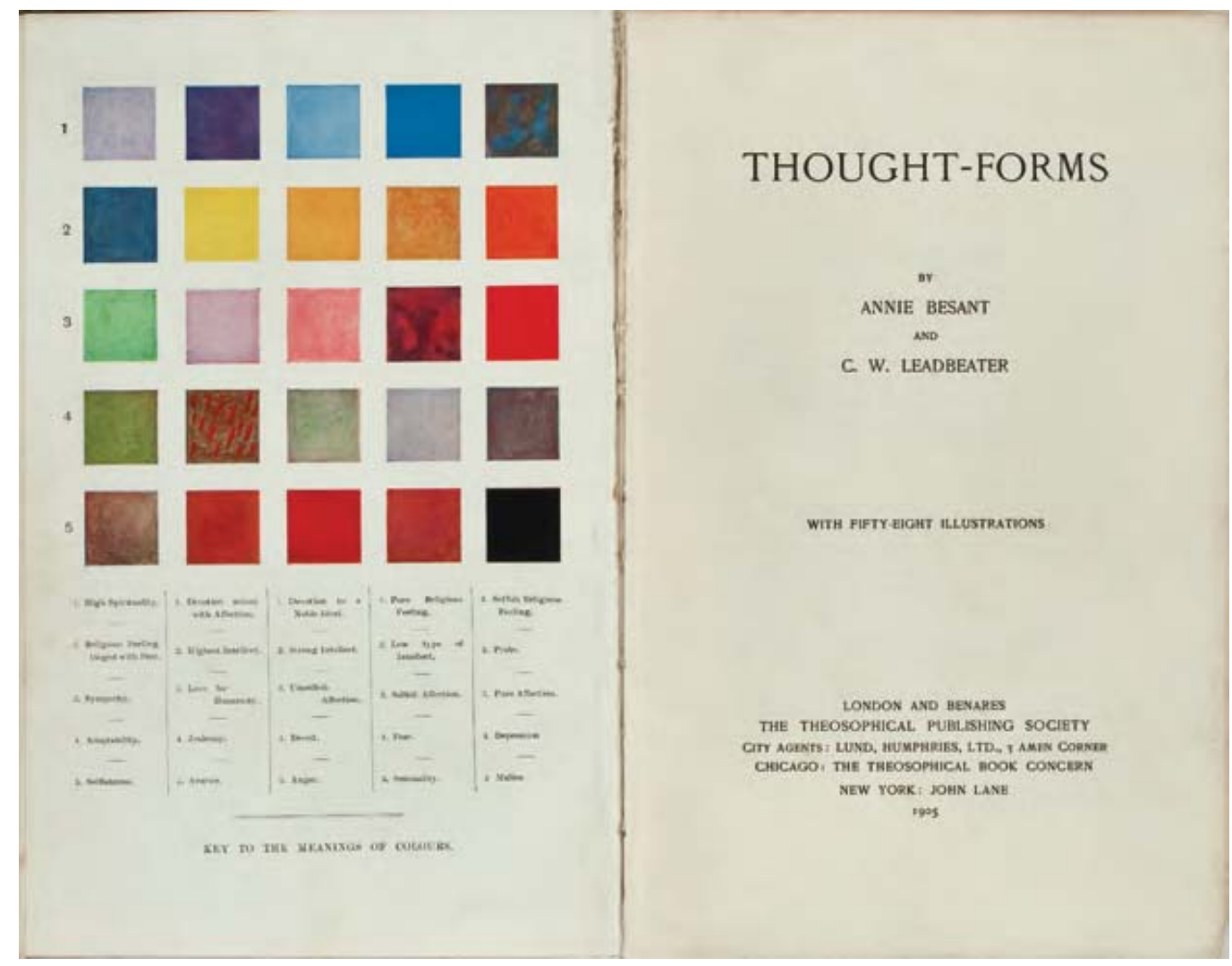

Abb. 11: Annie Besant und Charles Leadbeater: Thought Forms. London 1905, Frontispiz

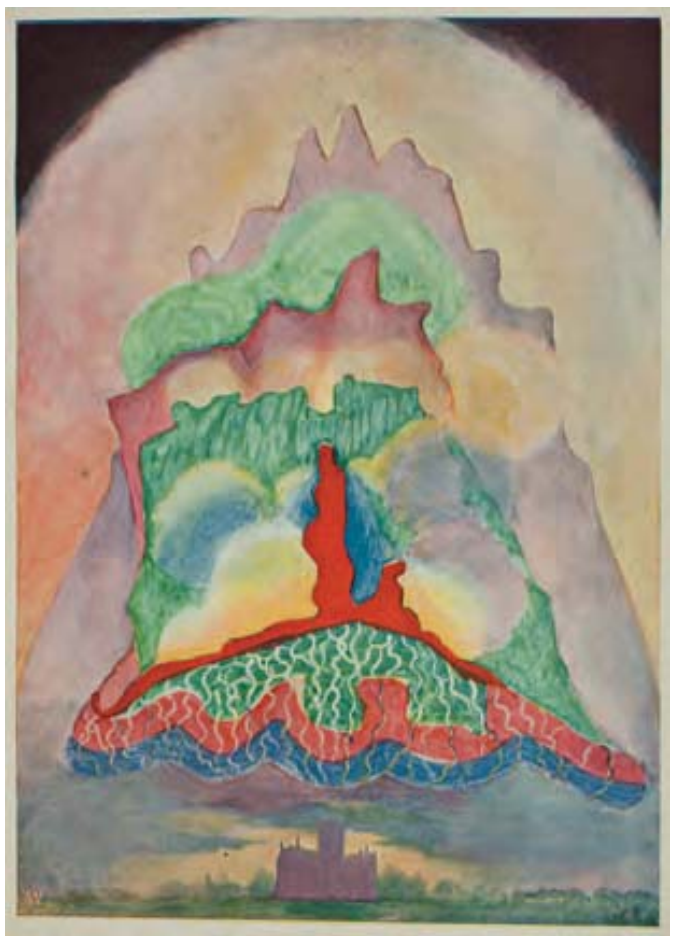

Abb. 12: Musik von Wagner. Aus: Annie Besant und Charles Leadbeater: Thought Forms, 1905 